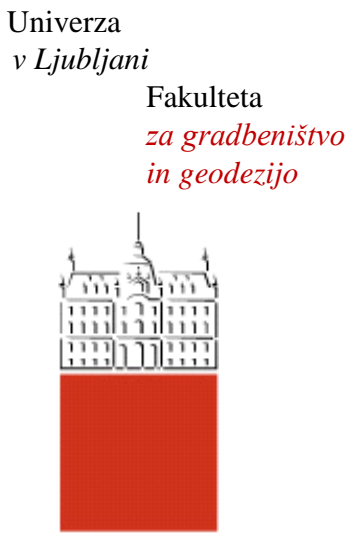

Jamova cesta 2

1000 Ljubljana, Slovenija

http://www3.fgg.uni-lj.si/

\section{DRUGG - Digitalni repozitorij UL FGG http://drugg.fgg.uni-lj.si/}

Ta članek je avtorjeva zadnja recenzirana različica, kot je bila sprejeta po opravljeni recenziji.

Prosimo, da se pri navajanju sklicujte na bibliografske podatke, kot je navedeno:
University
of Ljubljana

Faculty of Civil and Geodetic Engineering

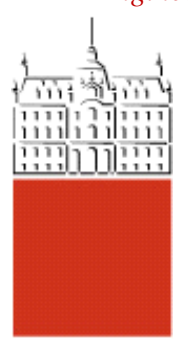

Jamova cesta 2 SI - 1000 Ljubljana, Slovenia http://www3.fgg.uni-lj.si/en/

DRUGG - The Digital Repository http://drugg.fgg.uni-lj.si/

This version of the article is author's manuscript as accepted for publishing after the review process.

When citing, please refer to the publisher's bibliographic information as follows:

Zupan, D., Saje, M. 2006. The linearized three-dimensional beam theory of naturally curved and twisted beams: the strain vectors formulation. Computer Methods in Applied Mechanics and Engineering 195,33-36: 4557-4578. DOI: 10.1016/j.cma.2005.10.002. 


\title{
The linearized three-dimensional beam theory of naturally curved and twisted beams: the strain vectors formulation
}

\author{
D. Zupan* and M. Saje \\ University of Ljubljana, Faculty of Civil and Geodetic Engineering, Jamova 2, \\ SI-1115 Ljubljana, Slovenia
}

\begin{abstract}
This paper presents the equations of the linearized geometrically exact three-dimensional beam theory of naturally curved and twisted beams. A new finite-element formulation for the linearized theory is proposed in which the strain vectors are the only unknown functions. The linear form of the consistency condition that the equilibrium and the constitutive internal force and moment vectors are equal, is enforced to be satisfied at chosen points. An arbitrary curved and twisted axis of the beam is taken into account which demands proper consideration of the non-linearity of spatial rotations. The accuracy and the efficiency of the derived numerical algorithm are demonstrated by comparing present numerical results with various analytical and numerical results.
\end{abstract}

Key words: linear beam theory, naturally curved and twisted beam, consistent linearization, strain measure, three-dimensional beam, three-dimensional rotation

\section{Introduction}

Initially curved and/or twisted beams are often used in buildings, bridges and mechanical devices as basic bearing elements. That is why the mathematical modelling of their behaviour under the mechanical and thermal loads has been a subject of research both in past and at present, see, e.g. the recent publications by Atanackovic and Glavardanov [1], Cho and Lee [3], Ganapathi et al. [5], Kulikov and Plotnikova [9], Leung [10], Madhusudhana et al. [12], Tang and $\mathrm{Yu}$ [23], $\mathrm{Yu}$ and Fang [27].

One of the well-known initially curved and twisted beam formulations is the so called 'geometrically exact finite-strain beam theory' (Reissner [15] and Simo

* Corresponding author. E-mail addresss: dejan.zupan@fgg.uni-lj.si 
[18]). Several finite-element formulations have been proposed for the numerical solution of its governing equations, see, e.g. Ibrahimbegovic [6], Jelenić and Saje [8], Schulz and Filippou [17], Simo and Vu-Quoc [19], Smoleński [20], to list just a few among the more often cited works.

The objective of the present paper is twofold: to derive the equations of the kinematically linearized finite-strain beam theory which assumes small displacements, rotations and strains but is capable of considering an arbitrary initial geometry and material behaviour, and to propose a stable, accurate and computationally efficient finite-element formulation for the solution of the linearized equations.

The linearized theory is deduced by a strict use of the Gateaux derivative on the non-linear functionals and by the proper consideration of the non-trivial linearization of the spatial rotations. In the numerical solution algorithm, we base our derivations on the vector of strain measures as the only unknown functions in a finite element. Such approach has proven to be efficient and allows us to satisfy easily the linear form of the consistency condition at the cross-section, that the stress-resultants, obtained from the equilibrium equations, are equal to those found from the constitutive equations.

An arbitrary finite-value initial bending and/or twisting curvature of the beam can be prescribed at the unloaded initial configuration, but only a sufficiently small loading is allowed to imply small displacements, rotations and strains. This type of assumptions is usually suggested in building design codes and followed in practical design by structural engineers.

\section{Geometry of the three-dimensional beam}

The geometrically exact finite-strain beam theory assumes that an arbitrary configuration of the beam is described by two independent vector fields (see Figure 1),

(i) the position vector $\vec{r}(x)$ of the line of centroids, and

(ii) the orthonormal base vectors $\left\{\vec{G}_{1}(x), \vec{G}_{2}(x), \vec{G}_{3}(x)\right\}$ spanning the planes of the cross-sections.

" $x$ " is the arc-length parameter of the line of centroids in the undeformed configuration. Unit vectors $\vec{G}_{2}(x)$ and $\vec{G}_{3}(x)$ point along the principal axes of inertia of the cross-section at $x$, and $\vec{G}_{1}$ is the cross-sectional unit normal: $\vec{G}_{1}=\vec{G}_{2} \times \vec{G}_{3}$. Note that $\vec{G}_{1}$ is generally not colinear with the tangent to the line of centroids, $\frac{d \vec{r}}{d x}$ (Figure 1 ). 


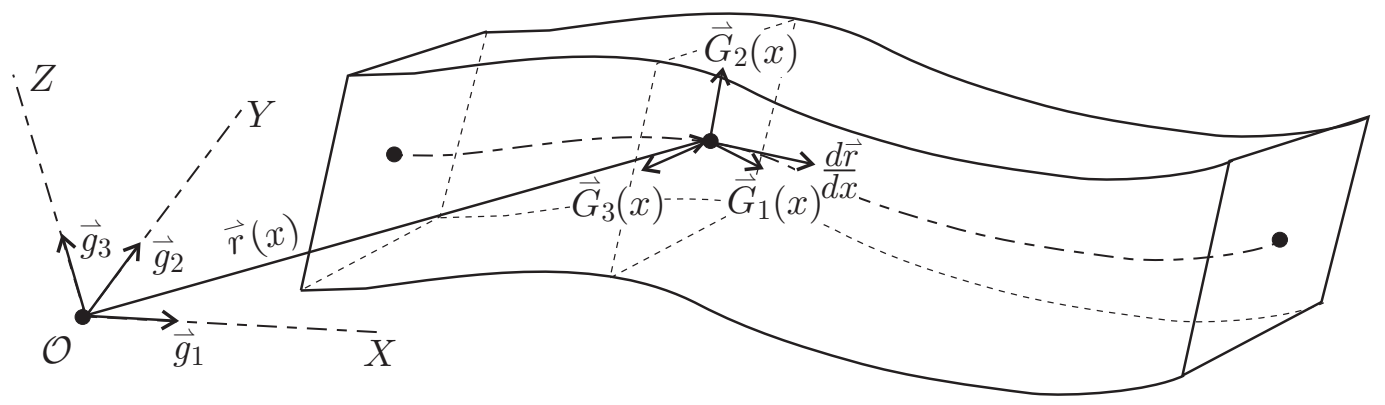

Fig. 1. Model of the three-dimensional beam.

In order to describe the deformation of the beam with respect to the physical space, which is the Euclidean linear vector space $\mathbb{R}^{3}$, we introduce a reference point $\mathcal{O}$ and a triad of fixed orthonormal vectors $\left\{\vec{g}_{1}, \vec{g}_{2}, \vec{g}_{3}\right\}$, which define the global coordinate system $(X, Y, Z)$. The position of a cross-section with respect to the global coordinate system is described by rotation matrix $\mathbf{R}$, being constructed so that its $i$-th column represents the components of the base vector $\vec{G}_{i}$ with respect to the fixed basis. In what follows we will have to express abstract vectors with respect to either of the two bases. Their components will be represented as one-column matrices, marked by a boldface font, and equipped with an index denoting the basis used; e.g., the matrix representation of the position vector $\vec{r}$ will be denoted by $\boldsymbol{r}_{g}$. Yet its matrix representation will still be termed the position vector. It is easy to show that the rotation matrix not only represents the physical rotation of a vector, but also the coordinate transformation between the components of a fixed vector with respect to the two bases

$$
\boldsymbol{v}_{g}=\mathbf{R} \boldsymbol{v}_{G}
$$

Only three scalar values suffice to describe the rotation in the three-dimensional space. There is a number of ways of choosing these parameters. Here we employ the three components of the rotational vector $\boldsymbol{\vartheta}_{g}[2]$ which is defined so that it lies on the axis of rotation and has the length equal to the angle of rotation. If we introduce a skew-symmetric matrix $\boldsymbol{\Theta}$

$$
\boldsymbol{\Theta}=\left[\begin{array}{ccc}
0 & -\vartheta_{3} & \vartheta_{2} \\
\vartheta_{3} & 0 & -\vartheta_{1} \\
-\vartheta_{2} & \vartheta_{1} & 0
\end{array}\right]
$$

composed from components $\left\{\vartheta_{1}, \vartheta_{2}, \vartheta_{3}\right\}$ of the vector $\boldsymbol{\vartheta}_{g}$, the rotation matrix is expressed by the Rodrigues formula

$$
\mathbf{R}=\mathbf{I}+\frac{\sin \vartheta}{\vartheta} \boldsymbol{\Theta}+\frac{1-\cos \vartheta}{\vartheta^{2}} \boldsymbol{\Theta}^{2},
$$


where $\mathbf{I}$ is the identity matrix, and $\vartheta=\left\|\boldsymbol{\vartheta}_{g}\right\|=\sqrt{\vartheta_{1}^{2}+\vartheta_{2}^{2}+\vartheta_{3}^{2}}$.

\section{Strain vectors}

The geometrically exact finite-strain beam theory introduces two strain vectors [15]: (i) the translational strain vector $\gamma_{G}$, and (ii) the rotational strain vector $\boldsymbol{\kappa}_{G}$. When expressed with respect to the base vectors $\vec{G}_{i}$, their components have physical interpretation: $\gamma_{G}(1)=\gamma_{1}$ is the extensional strain, and $\gamma_{G}(2)=\gamma_{2}, \gamma_{G}(3)=\gamma_{3}$ are shear strains; similarly, $\boldsymbol{\kappa}_{G}(1)=\kappa_{1}$ is the torsional strain, and $\kappa_{2}, \kappa_{3}$ are the bending strains (curvatures).

The relations between the strain, displacement and rotation vectors are derived from the condition that the strains and stresses are consistent with the virtual work principle for any internal forces and any magnitude of deformation. This condition yields the following relationships between the variations of kinematic vector variables $\left(\boldsymbol{r}_{g}, \boldsymbol{\vartheta}_{g}\right)$ and the variations of strain vectors $\left(\boldsymbol{\gamma}_{G}, \boldsymbol{\kappa}_{G}\right)$

$$
\begin{aligned}
\delta \boldsymbol{\gamma}_{G} & =\mathbf{R}^{T}\left(\delta \boldsymbol{r}_{g}^{\prime}-\delta \boldsymbol{\vartheta}_{g} \times \boldsymbol{r}_{g}^{\prime}\right) \\
\delta \boldsymbol{\kappa}_{G} & =\mathbf{R}^{T} \delta \boldsymbol{\vartheta}_{g}^{\prime}
\end{aligned}
$$

The prime (') denotes the derivative with respect to $x$, and " $\times$ " is the cross vector product. Equations (4) and (5) are sufficient for the derivation of the linear theory. For completeness we also give the integrated form of these equations (the details of the derivation were presented by Reissner [15] and Ibrahimbegovic [7])

$$
\begin{aligned}
\boldsymbol{\gamma}_{G} & =\mathbf{R}^{T} \boldsymbol{r}_{g}^{\prime}+\boldsymbol{c}_{G} \\
\boldsymbol{\kappa}_{G} & =\mathbf{T}^{T} \boldsymbol{\vartheta}_{g}^{\prime}+\boldsymbol{d}_{G},
\end{aligned}
$$

where

$$
\mathbf{T}^{T}=\mathbf{I}-\frac{1-\cos \vartheta}{\vartheta^{2}} \boldsymbol{\Theta}+\frac{\vartheta-\sin \vartheta}{\vartheta^{3}} \boldsymbol{\Theta}^{2}
$$

Vector functions $\boldsymbol{c}_{G}(x)$ and $\boldsymbol{d}_{G}(x)$ are variational constants which we determine from the known strain and kinematic fields of the undeformed beam.

\subsection{Determination of variational constants when cross-sections are orthogo- nal to the beam axis}

When the cross-sections are orthogonal to the line of centroids at the undeformed configuration, we have $\gamma_{0, G}=\mathbf{0}$. Inserting the condition into (6) 
yields

$$
\boldsymbol{c}_{G}=-\mathbf{R}^{T}\left(\boldsymbol{\vartheta}_{0, g}\right) \boldsymbol{r}_{0, g}^{\prime}
$$

Because the cross-sections are perpendicular to the centroidal axis, its tangent vector, $\frac{d \vec{r}_{0}(x)}{d x}$, coincides with the normal vector of the cross-section:

$$
\vec{G}_{1}^{0}(x)=\frac{d \vec{r}_{0}(x)}{d x}
$$

By the use of the coordinate transformation (1), equation (9) can be restated in the matrix form as

$$
\boldsymbol{G}_{1}^{0}=\mathbf{R}_{0}^{T} \boldsymbol{r}_{0, g}^{\prime}
$$

The insertion in equation (8) gives

$$
\boldsymbol{c}_{G}=-\mathbf{R}_{0}^{T} \boldsymbol{r}_{0, g}^{\prime}=-\boldsymbol{G}_{1}^{0}=-\left[\begin{array}{l}
1 \\
0 \\
0
\end{array}\right] .
$$

For the variational constant, $\boldsymbol{d}_{G}$, no such a simple formula exists generally. $\boldsymbol{d}_{G}$ is obtained from known rotations in the undeformed configuration $\left(\boldsymbol{\kappa}_{0, G}=\mathbf{0}\right)$ :

$$
\boldsymbol{d}_{G}=-\mathbf{T}_{0}^{T} \boldsymbol{\vartheta}_{0, g}^{\prime}
$$

Only in the special case of the straight beam, we have $\boldsymbol{d}_{G}=\mathbf{0}$.

\section{Equilibrium equations and stress resultants}

The equilibrium equations of an infinitesimal element of a beam are given by the following differential equations:

$$
\begin{aligned}
\boldsymbol{n}_{g}(x) & =-\boldsymbol{N}_{g}^{\prime}(x) \\
\boldsymbol{m}_{g}(x) & =-\boldsymbol{M}_{g}^{\prime}(x)-\boldsymbol{r}_{g}^{\prime}(x) \times \boldsymbol{N}_{g}(x)
\end{aligned}
$$

The two stress-resultants, the force $\boldsymbol{N}_{g}$ and the moment $\boldsymbol{M}_{g}$, depend both on the external distributed force and moment vectors $\boldsymbol{n}_{g}$ and $\boldsymbol{m}_{g}$ per unit of the undeformed length of the axis, and on the deformed shape of the axis, described by its position vector $\boldsymbol{r}_{g}(x)$. As $\boldsymbol{N}_{g}$ and $\boldsymbol{M}_{g}$ satisfy the equilibrium equations, they are termed the equilibrium force and moment. On the other hand, the stress resultants can also be determined via strain vectors $\gamma_{G}$ and 
$\boldsymbol{\kappa}_{G}$ using the constitutive equations

$$
\begin{aligned}
\boldsymbol{N}_{G}^{C} & =\mathcal{C}_{N}\left(\boldsymbol{\gamma}_{G}, \boldsymbol{\kappa}_{G}\right) \\
\boldsymbol{M}_{G}^{C} & =\mathcal{C}_{M}\left(\boldsymbol{\gamma}_{G}, \boldsymbol{\kappa}_{G}\right),
\end{aligned}
$$

which gives the so called constitutive force and moment, $\boldsymbol{N}_{G}^{C}$ and $\boldsymbol{M}_{G}^{C}$. The two kinds of stress-resultants should theoretically be equal. This is, however, not the case in the discretized solution, unless the condition is explicitly required to be satisfied. The inequality of equilibrium and constitutive stresses is typical in the displacement-based finite element formulations.

In order to avoid this inconsistency of the numerical solution, we here explicitly require the equality of the two kinds of forces and moments. In the matrix form, this requirement is represented by

$$
\begin{aligned}
\mathbf{R}(x) \boldsymbol{N}_{G}^{C}(x) & =\boldsymbol{N}_{g}(x) \\
\mathbf{R}(x) \boldsymbol{M}_{G}^{C}(x) & =\boldsymbol{M}_{g}(x) .
\end{aligned}
$$

A further discussion and an application of the consistency conditions in the elastic-plastic finite element analysis of plane frames is presented in the paper by Vratanar and Saje [24].

\section{Governing equations}

The complete set of the beam equations consists of the consistency conditions

(15)-(16), the kinematic equations (6)-(7) and the equilibrium equations (11)(12):

$$
\begin{aligned}
\mathbf{R}(x) \boldsymbol{N}_{G}^{C}(x)-\boldsymbol{N}_{g}(x) & =\mathbf{0} \\
\mathbf{R}(x) \boldsymbol{M}_{G}^{C}(x)-\boldsymbol{M}_{g}(x) & =\mathbf{0} \\
\boldsymbol{N}_{g}^{\prime}(x)+\boldsymbol{n}_{g}(x) & =\mathbf{0} \\
\boldsymbol{M}_{g}^{\prime}(x)+\boldsymbol{m}_{g}(x)-\boldsymbol{N}_{g}(x) \times \mathbf{R}(x)\left(\boldsymbol{\gamma}_{G}(x)-\boldsymbol{c}_{G}(x)\right) & =\mathbf{0} \\
\boldsymbol{r}_{g}^{\prime}(x)-\mathbf{R}(x)\left(\boldsymbol{\gamma}_{G}(x)-\boldsymbol{c}_{G}(x)\right) & =\mathbf{0} \\
\boldsymbol{\vartheta}_{g}^{\prime}(x)-\mathbf{T}^{-T}(x)\left(\boldsymbol{\kappa}_{G}(x)-\boldsymbol{d}_{G}(x)\right) & =\mathbf{0} .
\end{aligned}
$$

In (20) $\boldsymbol{r}_{g}^{\prime}$ has been replaced by $\mathbf{R}\left(\boldsymbol{\gamma}_{G}-\boldsymbol{c}_{G}\right)$. The related boundary conditions at the two boundaries, $x=0$ and $x=L$, are

$$
\begin{array}{ll}
\boldsymbol{N}_{g}(0)=\boldsymbol{S}_{g}^{0} & \boldsymbol{N}_{g}(L)=\boldsymbol{S}_{g}^{L} \\
\boldsymbol{M}_{g}(0)=\boldsymbol{P}_{g}^{0} & \boldsymbol{M}_{g}(L)=\boldsymbol{P}_{g}^{L} .
\end{array}
$$

$\boldsymbol{S}_{g}^{0}, \boldsymbol{P}_{g}^{0}, \boldsymbol{S}_{g}^{L}, \boldsymbol{P}_{g}^{L}$ are the external boundary point loads and moments. 
Equations (19)-(22) constitute the system of four first-order ordinary matrix differential equations. When $\boldsymbol{n}_{g}, \boldsymbol{m}_{g}, \boldsymbol{\gamma}_{G}$ and $\boldsymbol{\kappa}_{G}$ are given analytic functions of $x$, the formal solutions of these equations read

$$
\begin{aligned}
\boldsymbol{N}_{g}(x) & =\boldsymbol{N}_{g}(0)-\int_{0}^{x} \boldsymbol{n}_{g}(\xi) d \xi \\
\boldsymbol{M}_{g}(x) & =\boldsymbol{M}_{g}(0)+\int_{0}^{x}\left[\boldsymbol{N}_{g}(\xi) \times \mathbf{R}(\xi)\left(\boldsymbol{\gamma}_{G}(\xi)-\boldsymbol{c}_{G}(\xi)\right)-\boldsymbol{m}_{g}(\xi)\right] d \xi \\
\boldsymbol{r}_{g}(x) & =\boldsymbol{r}_{g}^{0}+\int_{0}^{x} \mathbf{R}(\xi)\left(\boldsymbol{\gamma}_{G}(\xi)-\boldsymbol{c}_{G}(\xi)\right) d \xi \\
\boldsymbol{\vartheta}_{g}(x) & =\boldsymbol{\vartheta}_{g}^{0}+\int_{0}^{x} \mathbf{T}^{-T}\left(\boldsymbol{\vartheta}_{g}(\xi)\right)\left(\boldsymbol{\kappa}_{G}(\xi)-\boldsymbol{d}_{G}(\xi)\right) d \xi
\end{aligned}
$$

Unfortunately, the integrals in equations (26)-(28) cannot be obtained analytically. We show later that the linearized theory needs only the weak forms of (27)-(28) to be employed, which simplifies the matter substantially.

The fulfilment of the displacement and rotation boundary conditions at $x=L$ places additional requirements on strain vectors:

$$
\begin{gathered}
\boldsymbol{r}_{g}(L)-\boldsymbol{r}_{g}^{0}-\int_{0}^{L} \mathbf{R}(\xi)\left(\gamma_{G}(\xi)-\boldsymbol{c}_{G}(\xi)\right) d \xi=\mathbf{0} \\
\boldsymbol{\vartheta}_{g}(L)-\boldsymbol{\vartheta}_{g}^{0}-\int_{0}^{L} \mathbf{T}^{-T}\left(\boldsymbol{\vartheta}_{g}(\xi)\right)\left(\boldsymbol{\kappa}_{G}(\xi)-\boldsymbol{d}_{G}(\xi)\right) d \xi=\mathbf{0} .
\end{gathered}
$$

The complete set of the equations of the geometrically exact three-dimensional beam then consists of the algebraic equations (17) and (18), the kinematic boundary conditions (29) and (30), and the static boundary conditions (23) and (24):

$$
\begin{aligned}
& \boldsymbol{f}_{1}=\mathbf{R} \boldsymbol{N}_{G}^{C}(x)-\boldsymbol{N}_{g}(x)=\mathbf{0} \\
& \boldsymbol{f}_{2}=\mathbf{R} \boldsymbol{M}_{G}^{C}(x)-\boldsymbol{M}_{g}(x)=\mathbf{0} \\
& \boldsymbol{f}_{3}=\boldsymbol{r}_{g}^{L}-\boldsymbol{r}_{g}^{0}-\int_{0}^{L} \mathbf{R}\left(\boldsymbol{\gamma}_{G}-\boldsymbol{c}_{G}\right) d x=\mathbf{0} \\
& \boldsymbol{f}_{4}=\boldsymbol{\vartheta}_{g}^{L}-\boldsymbol{\vartheta}_{g}^{0}-\int_{0}^{L} \mathbf{T}^{-T}\left(\boldsymbol{\vartheta}_{g}\right)\left(\boldsymbol{\kappa}_{G}-\boldsymbol{d}_{G}\right) d x=\mathbf{0} \\
& \boldsymbol{f}_{5}=\boldsymbol{S}_{g}^{0}+\boldsymbol{N}_{g}^{0}=\mathbf{0} \\
& \boldsymbol{f}_{6}=\boldsymbol{P}_{g}^{0}+\boldsymbol{M}_{g}^{0}=\mathbf{0} \\
& \boldsymbol{f}_{7}=\boldsymbol{S}_{g}^{L}-\boldsymbol{N}_{g}^{0}+\int_{0}^{L} \boldsymbol{n}_{g} d x=\mathbf{0} \\
& \boldsymbol{f}_{8}=\boldsymbol{P}_{g}^{L}-\boldsymbol{M}_{g}^{0}-\int_{0}^{L}\left[\boldsymbol{N}_{g} \times \mathbf{R}\left(\boldsymbol{\gamma}_{G}-\boldsymbol{c}_{G}\right)-\boldsymbol{m}_{g}\right] d x=\mathbf{0} .
\end{aligned}
$$

Equations (31)-(38) along with the auxiliary relations (25)-(28) and (13)- 
(14) constitute the set of eight equations for eight unknowns: (i) boundary kinematic vectors $\boldsymbol{r}_{g}^{0}, \boldsymbol{\vartheta}_{g}^{0}, \boldsymbol{r}_{g}^{L}, \boldsymbol{\vartheta}_{g}^{L}$, (ii) boundary equilibrium stress resultants $\boldsymbol{N}_{g}^{0}, \boldsymbol{M}_{g}^{0}$, and (iii) strain vector functions $\boldsymbol{\gamma}_{G}(x)$ and $\boldsymbol{\kappa}_{G}(x)$ along the beam.

The system of equations (31)-(38) is non-linear and must be solved iteratively. In practical design of structures only small displacements and rotations are allowed. For this class of problems, it is reasonable to assume that the linearized equations give sufficiently accurate results. The linearization is described in the next section.

\section{Linearized equations of the beam}

\subsection{Linearization of a scalar functional}

Mathematically, equations (31)-(38) introduce 24 scalar functionals, dependent on the (primary) unknowns $\boldsymbol{r}_{g}^{0}, \boldsymbol{\vartheta}_{g}^{0}, \boldsymbol{N}_{g}^{0}, \boldsymbol{M}_{g}^{0}, \boldsymbol{r}_{g}^{L}, \boldsymbol{\vartheta}_{g}^{L}, \boldsymbol{\gamma}_{G}(x)$, and $\boldsymbol{\kappa}_{G}(x)$. Let $\boldsymbol{y}$ denote the entire vector of unknowns. The linearization of a scalar functional $\mathcal{F}(\boldsymbol{y})$ requires first finding the variation of the functional at $\boldsymbol{y}$ in the direction $\delta \boldsymbol{y}$ in the Gâteaux sense [26]:

$$
\delta \mathcal{F}(\boldsymbol{y} ; \delta \boldsymbol{y})=\lim _{\alpha \rightarrow 0} \frac{\mathcal{F}(\boldsymbol{y}+\alpha \delta \boldsymbol{y})-\mathcal{F}(\boldsymbol{y})}{\alpha}=\left.\frac{d}{d \alpha}\right|_{\alpha=0} \mathcal{F}(\boldsymbol{y}+\alpha \delta \boldsymbol{y}) .
$$

The linearization of the functional $\mathcal{F}(\boldsymbol{y})$ about an arbitrary value $\boldsymbol{y}=\boldsymbol{y}_{0}$ is defined by

$$
\mathcal{F}\left(\boldsymbol{y}_{0}+\delta \boldsymbol{y}\right)=\mathcal{F}\left(\boldsymbol{y}_{0}\right)+\delta \mathcal{F}\left(\boldsymbol{y}_{0} ; \delta \boldsymbol{y}\right) .
$$

By setting $\mathcal{F}\left(\boldsymbol{y}_{0}+\delta \boldsymbol{y}\right)=0$, we obtain

$$
\delta \mathcal{F}\left(\boldsymbol{y}_{0} ; \delta \boldsymbol{y}\right)=-\mathcal{F}\left(\boldsymbol{y}_{0}\right),
$$

which is the linearization of the equation $\mathcal{F}(\boldsymbol{y})=0$ about $\boldsymbol{y}_{0}$. Now, since we have already introduced a 'coordinate system', the variation can be further worked out and replaced by the sum of partial derivatives with respect to the components of $\boldsymbol{y}=\left(y_{1}, y_{2}, \ldots, y_{n}\right)$ :

$$
\delta \mathcal{F}\left(\boldsymbol{y}_{0} ; \delta \boldsymbol{y}\right)=\left.\sum_{j=1}^{n} \frac{\partial \mathcal{F}}{\partial y_{j}}\right|_{\boldsymbol{y}_{0}} \delta y_{j}
$$

The linearization of the vector functional, $\mathcal{F}(\boldsymbol{y})=\left(\mathcal{F}_{1}(\boldsymbol{y}), \mathcal{F}_{2}(\boldsymbol{y}), \ldots, \mathcal{F}_{n}(\boldsymbol{y})\right)$, is obtained in an analogous way as

$$
\delta \mathcal{F}_{i}\left(\boldsymbol{y}_{0} ; \delta \boldsymbol{y}\right)=\left.\sum_{j=1}^{n} \frac{\partial \mathcal{F}_{i}}{\partial y_{j}}\right|_{\boldsymbol{y}_{0}} \delta y_{j}, \quad i=1,2, \ldots, n
$$


This equation holds in the linear vector spaces. The linearization of a functional in non-linear spaces requires a somewhat different procedure which is briefly described in the following subsection.

\subsection{Linearization of the rotation matrix}

Because the rotation matrix $\mathbf{R}$ is expressed in terms of the non-additive rotational vector $\boldsymbol{\vartheta}_{g}$ (see (3)) (that is why it is often called a 'pseudo-vector'), the linearization of the rotation matrix requires a special treatment. If the change of $\boldsymbol{\vartheta}_{g}$ is denoted by $\alpha \delta \boldsymbol{\vartheta}_{g}$, the rotational operator at the perturbed value of its argument is the product $\mathbf{R}\left(\alpha \delta \boldsymbol{\vartheta}_{g}\right) \mathbf{R}\left(\boldsymbol{\vartheta}_{g}\right)$, so that the difference between the original and the perturbed value of the rotational operator is

$$
\Delta \mathbf{R}=\mathbf{R}\left(\alpha \delta \boldsymbol{\vartheta}_{g}\right) \mathbf{R}\left(\boldsymbol{\vartheta}_{g}\right)-\mathbf{R}\left(\boldsymbol{\vartheta}_{g}\right)
$$

The Gâteaux variation of $\mathbf{R}$ then follows from the definition

$$
\delta \mathbf{R}=\left.\frac{d}{d \alpha}\right|_{\alpha=0} \mathbf{R}\left(\alpha \delta \boldsymbol{\vartheta}_{g}\right) \mathbf{R}\left(\boldsymbol{\vartheta}_{g}\right)
$$

From (2) and (3) we have

$$
\mathbf{R}\left(\alpha \delta \boldsymbol{\vartheta}_{g}\right)=\mathbf{I}+\frac{\sin (\alpha \delta \vartheta)}{\alpha \delta \vartheta} \alpha \delta \boldsymbol{\Theta}+\frac{1-\cos (\alpha \delta \vartheta)}{(\alpha \delta \vartheta)^{2}}(\alpha \delta \boldsymbol{\Theta})^{2}
$$

where

$$
\delta \boldsymbol{\Theta}=\left[\begin{array}{ccc}
0 & -\delta \vartheta_{3} & \delta \vartheta_{2} \\
\delta \vartheta_{3} & 0 & -\delta \vartheta_{1} \\
-\delta \vartheta_{2} & \delta \vartheta_{1} & 0
\end{array}\right]
$$

$\delta \boldsymbol{\Theta}$ is the skew-symmetric matrix, whose components are formed from the components $\left\{\delta \vartheta_{1}, \delta \vartheta_{2}, \delta \vartheta_{3}\right\}$ of its axial vector $\delta \vartheta_{g}$. After taking the derivative with respect to $\alpha$ and evaluating the result at $\alpha=0$, equation (42) gives

$$
\delta \mathbf{R}=\delta \boldsymbol{\Theta} \mathbf{R}
$$

For the further simplification of the result, we will introduce the skew-symmetric matrix $\mathbf{S}(\boldsymbol{v})$ formed from the components of vector $\boldsymbol{v}$. Then the cross vector product, $\boldsymbol{v} \times \boldsymbol{u}$, between arbitrary two vectors $\boldsymbol{v}$ and $\boldsymbol{u}$ can be written as the 
matrix product of $\mathbf{S}(\boldsymbol{v})$ and $\boldsymbol{u}$

$$
\boldsymbol{v} \times \boldsymbol{u}=\left[\begin{array}{c}
v_{2} u_{3}-v_{3} u_{2} \\
v_{3} u_{1}-v_{1} u_{3} \\
v_{1} u_{2}-v_{2} u_{1}
\end{array}\right]=\left[\begin{array}{ccc}
0 & -v_{3} & v_{2} \\
v_{3} & 0 & -v_{1} \\
-v_{2} & v_{1} & 0
\end{array}\right]\left[\begin{array}{l}
u_{1} \\
u_{2} \\
u_{3}
\end{array}\right]=\mathbf{S}(\boldsymbol{v}) \boldsymbol{u} .
$$

When multiplied by an arbitrary vector, $\boldsymbol{u}$, equation (43) gives

$$
\delta \mathbf{R} \boldsymbol{u}=\delta \boldsymbol{\Theta} \mathbf{R} \boldsymbol{u}=\mathbf{S}\left(\boldsymbol{\delta} \vartheta_{g}\right) \mathbf{R} \boldsymbol{u}=\delta \boldsymbol{\vartheta}_{g} \times \mathbf{R} \boldsymbol{u}
$$

Taking into account that $\boldsymbol{v} \times \boldsymbol{u}=-\boldsymbol{u} \times \boldsymbol{v}$ and considering (44) we get

$$
\delta \mathbf{R} \boldsymbol{u}=\delta \boldsymbol{\vartheta}_{g} \times \mathbf{R} \boldsymbol{u}=-\mathbf{R} \boldsymbol{u} \times \delta \boldsymbol{\vartheta}_{g}=-\mathbf{S}(\mathbf{R} \boldsymbol{u}) \delta \boldsymbol{\vartheta}_{g}
$$

\subsection{Linearization of equations of the beam}

Equations (31)-(38) will be varied at $\boldsymbol{r}_{g}^{0}, \boldsymbol{\vartheta}_{g}^{0}, \boldsymbol{N}_{g}^{0}, \boldsymbol{M}_{g}^{0}, \boldsymbol{r}_{g}^{L}, \boldsymbol{\vartheta}_{g}^{L}, \boldsymbol{\gamma}_{G}(x), \boldsymbol{\kappa}_{G}(x)$ in 'directions' $\delta \boldsymbol{r}_{g}^{0}, \delta \boldsymbol{\vartheta}_{g}^{0}, \delta \boldsymbol{N}_{g}^{0}, \delta \boldsymbol{M}_{g}^{0}, \delta \boldsymbol{r}_{g}^{L}, \delta \boldsymbol{\vartheta}_{g}^{L}, \delta \boldsymbol{\gamma}_{G}(x)$, and $\delta \boldsymbol{\kappa}_{G}(x)$. The deduction of the variations of the equations is simplified if variations of some of the quantities are prepared in advance.

Function $\boldsymbol{N}_{g}(x)$ depends on $\boldsymbol{N}_{g}^{0}$ and $\boldsymbol{n}_{g}(x)$. When the loading is deformationindependent, which is the case in the present analysis, $\boldsymbol{n}_{g}(x)$ does not depend on the unknown functions, and so

$$
\delta \boldsymbol{N}_{g}(x)=\delta \boldsymbol{N}_{g}^{0}
$$

The variation of the derivative of the rotational vector, $\boldsymbol{\vartheta}_{g}^{\prime}$, is given by equation $(5)$ :

$$
\delta \boldsymbol{\vartheta}_{g}^{\prime}=\mathbf{R} \delta \boldsymbol{\kappa}_{G} .
$$

The integration of equation (47) with respect to $x$ gives

$$
\delta \boldsymbol{\vartheta}_{g}(x)=\delta \boldsymbol{\vartheta}_{g}^{0}+\int_{0}^{x} \mathbf{R}(\xi) \delta \boldsymbol{\kappa}_{G}(\xi) d \xi
$$

When (48) is inserted into (45) we obtain

$$
\delta \mathbf{R} \boldsymbol{u}=-\mathbf{S}(\mathbf{R} \boldsymbol{u}) \delta \boldsymbol{\vartheta}_{g}^{0}-\mathbf{S}(\mathbf{R} \boldsymbol{u}) \int_{0}^{x} \mathbf{R}(\xi) \delta \boldsymbol{\kappa}_{G}(\xi) d \xi
$$

this holds true for any vector $\boldsymbol{u}$. 
Varying $\boldsymbol{M}_{g}(x)$ as expressed by (26), and employing (46) and (49) gives

$$
\begin{aligned}
\delta \boldsymbol{M}_{g}(x)= & \delta \boldsymbol{M}_{g}^{0}+\int_{0}^{x}\left[\delta \boldsymbol{N}_{g} \times \mathbf{R}\left(\boldsymbol{\gamma}_{G}-\boldsymbol{c}_{G}\right)\right] d \xi+\int_{0}^{x}\left[\boldsymbol{N}_{g} \times \delta \mathbf{R}\left(\boldsymbol{\gamma}_{G}-\boldsymbol{c}_{G}\right)\right] d \xi \\
& +\int_{0}^{x}\left[\boldsymbol{N}_{g} \times \mathbf{R} \delta \boldsymbol{\gamma}_{G}\right] d \xi \\
= & \delta \boldsymbol{M}_{g}^{0}+\delta \boldsymbol{N}_{g}^{0} \times \int_{0}^{x} \mathbf{R}\left(\boldsymbol{\gamma}_{G}-\boldsymbol{c}_{G}\right) d \xi \\
& -\int_{0}^{x}\left[\boldsymbol{N}_{g} \times \mathbf{S}\left(\mathbf{R}\left(\boldsymbol{\gamma}_{G}-\boldsymbol{c}_{G}\right)\right) \delta \boldsymbol{\vartheta}_{g}^{0}\right] d \xi+\int_{0}^{x}\left[\boldsymbol{N}_{g} \times \mathbf{R} \delta \boldsymbol{\gamma}_{G}\right] d \xi \\
& -\int_{0}^{x}\left[\boldsymbol{N}_{g} \times \mathbf{S}\left(\mathbf{R}\left(\boldsymbol{\gamma}_{G}-\boldsymbol{c}_{G}\right)\right) \int_{0}^{\xi} \mathbf{R} \delta \boldsymbol{\kappa}_{G} d \eta\right] d \xi \\
= & \delta \boldsymbol{M}_{g}^{0}-\mathbf{S}\left(\int_{0}^{x} \mathbf{R}\left(\boldsymbol{\gamma}_{G}-\boldsymbol{c}_{G}\right) d \xi\right) \delta \boldsymbol{N}_{g}^{0} \\
& -\int_{0}^{x} \mathbf{S}\left(\boldsymbol{N}_{g}\right) \mathbf{S}\left(\mathbf{R}\left(\boldsymbol{\gamma}_{G}-\boldsymbol{c}_{G}\right)\right) d \xi \delta \boldsymbol{\vartheta}_{g}^{0}+\int_{0}^{x} \mathbf{S}\left(\boldsymbol{N}_{g}\right) \mathbf{R} \delta \boldsymbol{\gamma}_{G} d \xi \quad(50) \\
& -\int_{0}^{x} \mathbf{S}\left(\boldsymbol{N}_{g}\right) \mathbf{S}\left(\mathbf{R}\left(\boldsymbol{\gamma}_{G}-\boldsymbol{c}_{G}\right)\right) \int_{0}^{\xi} \mathbf{R} \delta \boldsymbol{\kappa}_{G} d \eta d \xi
\end{aligned}
$$

The linearization of the constitutive equations gives

$$
\begin{aligned}
\delta \boldsymbol{N}_{G}^{C} & =\delta \mathcal{C}^{N}=\mathbf{C}_{\gamma \gamma} \delta \boldsymbol{\gamma}_{G}+\mathbf{C}_{\gamma \kappa} \delta \boldsymbol{\kappa}_{G} \\
\delta \boldsymbol{M}_{G}^{C} & =\delta \mathcal{C}^{M}=\mathbf{C}_{\kappa \gamma} \delta \boldsymbol{\gamma}_{G}+\mathbf{C}_{\kappa \kappa} \delta \boldsymbol{\kappa}_{G} .
\end{aligned}
$$

Here the components of matrices $\mathbf{C}_{\gamma \gamma}, \mathbf{C}_{\gamma \kappa}, \mathbf{C}_{\kappa \gamma}$, and $\mathbf{C}_{\kappa \kappa}$ are the partial derivatives of $\mathcal{C}^{N}$ and $\mathcal{C}^{M}$ with respect to the components of $\boldsymbol{\gamma}_{G}$ and $\boldsymbol{\kappa}_{G}$ :

$$
\begin{array}{ll}
\mathbf{C}_{\gamma \gamma}=\left[\frac{\partial \mathcal{C}_{i}^{N}}{\partial \gamma_{j}}\right], & \mathbf{C}_{\gamma \kappa}=\left[\frac{\partial \mathcal{C}_{i}^{N}}{\partial \kappa_{j}}\right] \\
\mathbf{C}_{\kappa \gamma}=\left[\frac{\partial \mathcal{C}_{i}^{M}}{\partial \gamma_{j}}\right], & \mathbf{C}_{\kappa \kappa}=\left[\frac{\partial \mathcal{C}_{i}^{M}}{\partial \kappa_{j}}\right] .
\end{array}
$$

The matrix $\mathbf{C}=\left[\begin{array}{ll}\mathbf{C}_{\gamma \gamma} & \mathbf{C}_{\gamma \kappa} \\ \mathbf{C}_{\kappa \gamma} & \mathbf{C}_{\kappa \kappa}\end{array}\right]$ is called the cross-section constitutive tangent matrix.

After these preparations have been completed, the variations of the equations of the beam are easily derived and are as follows:

$$
\begin{aligned}
\delta \boldsymbol{f}_{1}(x) & =\delta \mathbf{R}(x) \boldsymbol{N}_{G}^{C}(x)+\mathbf{R}(x) \delta \boldsymbol{N}_{G}^{C}(x)-\delta \boldsymbol{N}_{g}(x) \\
\delta \boldsymbol{f}_{2}(x) & =\delta \mathbf{R}(x) \boldsymbol{M}_{G}^{C}(x)+\mathbf{R}(x) \delta \boldsymbol{M}_{G}^{C}(x)-\delta \boldsymbol{M}_{g}(x) \\
\delta \boldsymbol{f}_{3} & =\delta \boldsymbol{r}_{g}^{L}-\delta \boldsymbol{r}_{g}^{0}-\int_{0}^{L} \delta \mathbf{R}\left(\boldsymbol{\gamma}_{G}-\boldsymbol{c}_{G}\right) d x-\int_{0}^{L} \mathbf{R} \delta \boldsymbol{\gamma}_{G} d x
\end{aligned}
$$




$$
\begin{aligned}
\delta \boldsymbol{f}_{4} & =\delta \boldsymbol{\vartheta}_{g}^{L}-\delta \boldsymbol{\vartheta}_{g}^{0}-\int_{0}^{L} \mathbf{R} \delta \boldsymbol{\kappa}_{G} d x \\
\delta \boldsymbol{f}_{5} & =\delta \boldsymbol{N}_{g}^{0} \\
\delta \boldsymbol{f}_{6} & =\delta \boldsymbol{M}_{g}^{0} \\
\delta \boldsymbol{f}_{7} & =-\delta \boldsymbol{N}_{g}^{0} \\
\delta \boldsymbol{f}_{8} & =-\delta \boldsymbol{M}_{g}(L) .
\end{aligned}
$$

Only equation (56) needs some comments. Recall that the boundary condition in (34) is an integrated form of the kinematic equation (7). The variation of equation (7) is given by equation (5). By integrating (5) with respect to $x$ and evaluating the result at $x=L$, we obtain the result in (56).

The substitution of relations (46), (49), (50), (51), and (52) into (53)-(60) yields the variations of all equations with respect to the variations of the primary unknowns:

$$
\begin{aligned}
\delta \boldsymbol{f}_{1}(x)= & -\mathbf{S}\left(\mathbf{R}(x) \boldsymbol{N}_{G}^{C}(x)\right) \delta \boldsymbol{\vartheta}_{g}^{0}-\mathbf{S}\left(\mathbf{R}(x) \boldsymbol{N}_{G}^{C}(x)\right) \int_{0}^{x} \mathbf{R}(\xi) \delta \boldsymbol{\kappa}_{G}(\xi) d \xi \\
& +\mathbf{R}(x) \mathbf{C}_{\gamma \gamma}(x) \delta \boldsymbol{\gamma}_{G}(x)+\mathbf{R}(x) \mathbf{C}_{\gamma \kappa}(x) \delta \boldsymbol{\kappa}_{G}(x)-\delta \boldsymbol{N}_{g}^{0} \\
\delta \boldsymbol{f}_{2}(x)= & -\mathbf{S}\left(\mathbf{R}(x) \boldsymbol{M}_{G}^{C}(x)\right) \delta \boldsymbol{\vartheta}_{g}^{0}-\mathbf{S}\left(\mathbf{R}(x) \boldsymbol{M}_{G}^{C}(x)\right) \int_{0}^{x} \mathbf{R}(\xi) \delta \boldsymbol{\kappa}_{G}(\xi) d \xi \\
& +\mathbf{R}(x) \mathbf{C}_{\kappa \gamma}(x) \delta \boldsymbol{\gamma}_{G}(x)+\mathbf{R}(x) \mathbf{C}_{\kappa \kappa}(x) \delta \boldsymbol{\kappa}_{G}(x)-\delta \boldsymbol{M}_{g}^{0} \\
& +\mathbf{S}\left(\int_{0}^{x} \mathbf{R}\left(\boldsymbol{\gamma}_{G}-\boldsymbol{c}_{G}\right) d \xi\right) \delta \boldsymbol{N}_{g}^{0}+\int_{0}^{x} \mathbf{S}\left(\boldsymbol{N}_{g}\right) \mathbf{S}\left(\mathbf{R}\left(\boldsymbol{\gamma}_{G}-\boldsymbol{c}_{G}\right)\right) d \xi \delta \boldsymbol{\vartheta}_{g}^{0} \\
& +\int_{0}^{x} \mathbf{S}\left(\boldsymbol{N}_{g}\right) \mathbf{S}\left(\mathbf{R}\left(\boldsymbol{\gamma}_{G}-\boldsymbol{c}_{G}\right)\right) \int_{0}^{\xi} \mathbf{R} \delta \boldsymbol{\kappa}_{G} d \eta d \xi-\int_{0}^{x} \mathbf{S}\left(\boldsymbol{N}_{g}\right) \mathbf{R} \delta \boldsymbol{\gamma}_{G} d \xi \\
\delta \boldsymbol{f}_{3}= & \delta \boldsymbol{r}_{g}^{L}-\delta \boldsymbol{r}_{g}^{0}+\int_{0}^{L} \mathbf{S}\left(\mathbf{R}\left(\boldsymbol{\gamma}_{G}-\boldsymbol{c}_{G}\right)\right) d x \delta \boldsymbol{\vartheta}_{g}^{0} \\
& +\int_{0}^{L} \mathbf{S}\left(\mathbf{R}\left(\boldsymbol{\gamma}_{G}-\boldsymbol{c}_{G}\right)\right) \int_{0}^{x} \mathbf{R} \delta \boldsymbol{\kappa}_{G} d \xi d x-\int_{0}^{L} \mathbf{R} \delta \boldsymbol{\gamma}_{G} d x \\
\delta \boldsymbol{f}_{4}= & \delta \boldsymbol{\vartheta}_{g}^{L}-\delta \boldsymbol{\vartheta}_{g}^{0}-\int_{0}^{L} \mathbf{R} \delta \boldsymbol{\kappa}_{G} d x \\
\delta \boldsymbol{f}_{5}= & \delta \boldsymbol{N}_{g}^{0} \\
\delta \boldsymbol{f}_{6}= & \delta \boldsymbol{M}_{g}^{0} \\
\delta \boldsymbol{f}_{7}= & -\delta \boldsymbol{N}_{g}^{0} \\
\delta \boldsymbol{f}_{8}= & \mathbf{S}\left(\int_{0}^{L} \mathbf{R}\left(\boldsymbol{\gamma}_{G}-\boldsymbol{c}_{G}\right) d x\right) \delta \boldsymbol{N}_{g}^{0}+\int_{0}^{L} \mathbf{S}\left(\boldsymbol{N}_{g}\right) \mathbf{S}\left(\mathbf{R}\left(\boldsymbol{\gamma}_{G}-\boldsymbol{c}_{G}\right)\right) d x \delta \boldsymbol{\vartheta}_{g}^{0} \\
& -\delta \boldsymbol{M}_{g}^{0}-\int_{0}^{L} \mathbf{S}\left(\boldsymbol{N}_{g}\right) \mathbf{R} \delta \boldsymbol{\gamma}_{G} d x \\
& +\int_{0}^{L} \mathbf{S}\left(\boldsymbol{N}_{g}\right) \mathbf{S}\left(\mathbf{R}\left(\boldsymbol{\gamma}_{G}-\boldsymbol{c}_{G}\right)\right) \int_{0}^{x} \mathbf{R} \delta \boldsymbol{\kappa}_{G} d \xi d x . \\
&
\end{aligned}
$$


The set of linearized equations for unknown $\delta \boldsymbol{y}$ then takes the form

$$
\delta \boldsymbol{f}_{i}\left(\boldsymbol{y}_{0} ; \delta \boldsymbol{y}\right)=-\boldsymbol{f}_{i}\left(\boldsymbol{y}_{0}\right), \quad i=1, \ldots, 8
$$

where $\boldsymbol{f}_{i}$ are given in equations (31)-(38) and evaluated at the initial configuration $\boldsymbol{y}_{0}$. We assume that the geometry of the beam and its strains in the initial configuration are arbitrary, yet kinematically exact, so that equations (33)-(34) are identically satisfied at $\boldsymbol{y}_{0}$ (i.e., $\left.\boldsymbol{f}_{3}\left(\boldsymbol{y}_{0}\right)=\boldsymbol{f}_{4}\left(\boldsymbol{y}_{0}\right)=\mathbf{0}\right)$. Such a beam is called a naturally strained (extended, curved and twisted) beam.

The inspection of equations (61)-(64) and (68) shows that the linearized equations (69) do not appear in the form of a linear combination of the primary unknowns $\delta \boldsymbol{\gamma}_{G}(x)$ and $\delta \boldsymbol{\kappa}_{G}(x)$, which is due to the multiplicative nature of the spatial rotations. This is resolved after the discretization of the primary unknowns is made as presented in Section 7.

\subsection{Cross-sections are orthogonal to the beam axis in the initial configuration}

Let us assume that in the initial, undeformed and unloaded configuration, the cross-sections are orthogonal to the line of centroids, and that the initial stress-resultant vectors at $x=0$ are zero: $\boldsymbol{N}_{g}^{0}=\boldsymbol{M}_{g}^{0}=\mathbf{0}$. Then $\boldsymbol{\gamma}_{G}=\mathbf{0}$ and $\boldsymbol{c}_{G}=\left[\begin{array}{lll}-1 & 0 & 0\end{array}\right]^{T}$ at the undeformed state (see Section 3.1), so that the term $\mathbf{R}\left(\boldsymbol{\gamma}_{G}-\boldsymbol{c}_{G}\right)$ equals to the first column of the rotation matrix, here denoted by $\mathbf{R}^{(1)}$. The skew-symmetric matrix $\mathbf{S}\left(\mathbf{R}^{(1)}\right)$ will be denoted by $\mathbf{R}_{\mathrm{S}}^{(1)}$. Then the variations read

$$
\begin{aligned}
\delta \boldsymbol{f}_{1}(x)= & -\delta \boldsymbol{N}_{g}^{0}+\mathbf{R}(x) \mathbf{C}_{\gamma \gamma}(x) \delta \boldsymbol{\gamma}_{G}(x)+\mathbf{R}(x) \mathbf{C}_{\gamma \kappa}(x) \delta \boldsymbol{\kappa}_{G}(x) \\
\delta \boldsymbol{f}_{2}(x)= & \left(\int_{0}^{x} \mathbf{S}\left(\boldsymbol{N}_{g}\right) \mathbf{R}_{\mathrm{S}}^{(1)} d \xi\right) \delta \boldsymbol{\vartheta}_{g}^{0}+\left(\int_{0}^{x} \mathbf{R}_{\mathrm{S}}^{(1)} d \xi\right) \delta \boldsymbol{N}_{g}^{0}-\delta \boldsymbol{M}_{g}^{0} \\
& +\mathbf{R}(x) \mathbf{C}_{\kappa \gamma}(x) \delta \boldsymbol{\gamma}_{G}(x)+\mathbf{R}(x) \mathbf{C}_{\kappa \kappa}(x) \delta \boldsymbol{\kappa}_{G}(x) \\
& +\int_{0}^{x} \mathbf{S}\left(\boldsymbol{N}_{g}\right) \mathbf{R}_{\mathrm{S}}^{(1)}\left(\int_{0}^{\xi} \mathbf{R} \delta \boldsymbol{\kappa}_{G} d \eta\right) d \xi-\int_{0}^{x} \mathbf{S}\left(\boldsymbol{N}_{g}\right) \mathbf{R} \delta \boldsymbol{\gamma}_{G} d \xi \\
\delta \boldsymbol{f}_{3}= & -\delta \boldsymbol{r}_{g}^{0}+\left(\int_{0}^{L} \mathbf{R}_{\mathrm{S}}^{(1)} d x\right) \delta \boldsymbol{\vartheta}_{g}^{0}+\delta \boldsymbol{r}_{g}^{L} \\
& -\int_{0}^{L} \mathbf{R} \delta \boldsymbol{\gamma}_{G} d x+\int_{0}^{L} \mathbf{R}_{\mathrm{S}}^{(1)}\left(\int_{0}^{x} \mathbf{R} \delta \boldsymbol{\kappa}_{G} d \xi\right) d x \\
\delta \boldsymbol{f}_{4}= & -\delta \boldsymbol{\vartheta}_{g}^{0}+\delta \boldsymbol{\vartheta}_{g}^{L}-\int_{0}^{L} \mathbf{R} \delta \boldsymbol{\kappa}_{G} d x \\
\delta \boldsymbol{f}_{5}= & \delta \boldsymbol{N}_{g}^{0} \\
\delta \boldsymbol{f}_{6}= & \delta \boldsymbol{M}_{g}^{0} \\
\delta \boldsymbol{f}_{7}= & -\delta \boldsymbol{N}_{g}^{0}
\end{aligned}
$$




$$
\begin{aligned}
\delta \boldsymbol{f}_{8}= & \left(\int_{0}^{L} \mathbf{S}\left(\boldsymbol{N}_{g}\right) \mathbf{R}_{\mathrm{S}}^{(1)} d x\right) \delta \boldsymbol{\vartheta}_{g}^{0}+\left(\int_{0}^{L} \mathbf{R}_{\mathrm{S}}^{(1)} d x\right) \delta \boldsymbol{N}_{g}^{0}-\delta \boldsymbol{M}_{g}^{0} \\
& +\int_{0}^{L} \mathbf{S}\left(\boldsymbol{N}_{g}\right) \mathbf{R}_{\mathrm{S}}^{(1)}\left(\int_{0}^{x} \mathbf{R} \delta \boldsymbol{\kappa}_{G} d \xi\right) d x-\int_{0}^{L} \mathbf{S}\left(\boldsymbol{N}_{g}\right) \mathbf{R} \delta \boldsymbol{\gamma}_{G} d x
\end{aligned}
$$

The related right-hand side vectors are

$$
\begin{aligned}
-\boldsymbol{f}_{1} & =\boldsymbol{N}_{g}(x)-\int_{0}^{x} \boldsymbol{n}_{g} d \xi \\
-\boldsymbol{f}_{2} & =\boldsymbol{M}_{g}(x)-\int_{0}^{x}\left[\left(\int_{0}^{\xi} \boldsymbol{n}_{g} d \eta\right) \times \mathbf{R}^{(1)}+\boldsymbol{m}_{g}\right] d \xi \\
-\boldsymbol{f}_{3} & =\mathbf{0} \\
-\boldsymbol{f}_{4} & =\mathbf{0} \\
-\boldsymbol{f}_{5} & =-\boldsymbol{S}_{g}^{0} \\
-\boldsymbol{f}_{6} & =-\boldsymbol{P}_{g}^{0} \\
-\boldsymbol{f}_{7} & =-\boldsymbol{S}_{g}^{L}-\int_{0}^{L} \boldsymbol{n}_{g} d x \\
-\boldsymbol{f}_{8} & =-\boldsymbol{P}_{g}^{L}-\int_{0}^{L}\left[\left(\int_{0}^{x} \boldsymbol{n}_{g} d \xi\right) \times \mathbf{R}^{(1)}+\boldsymbol{m}_{g}\right] d x=\mathbf{0} .
\end{aligned}
$$

You can see that the assumption that the cross-sections are orthogonal to the line of centroids in the undeformed configuration results in a substantial simplification of the linearized equations.

\subsection{Update procedure}

Let us assume that the change of the unknown vector

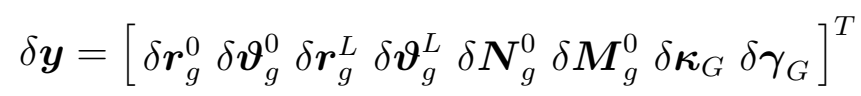

has been obtained from (69). In linear vector spaces, the improved value $\boldsymbol{y}$ is obtained by adding the change $\delta \boldsymbol{y}$ to the current value $\boldsymbol{y}_{0}$. The position vector, the stress-resultants, and the strain vectors are linear vectors; their changes are therefore simply added to the current approximation values:

$$
\begin{array}{cc}
\boldsymbol{r}_{g}^{0}=\boldsymbol{r}_{0, g}^{0}+\delta \boldsymbol{r}_{g}^{0} & \boldsymbol{r}_{g}^{L}=\boldsymbol{r}_{0, g}^{L}+\delta \boldsymbol{r}_{g}^{L} \\
\boldsymbol{N}_{g}^{0}=\boldsymbol{N}_{0, g}^{0}+\delta \boldsymbol{N}_{g}^{0} & \boldsymbol{M}_{g}^{0}=\boldsymbol{M}_{0, g}^{0}+\delta \boldsymbol{M}_{g}^{0} \\
\boldsymbol{\gamma}_{G}(x)=\gamma_{0, G}(x)+\delta \boldsymbol{\gamma}_{G}(x) \\
\boldsymbol{\kappa}_{G}(x)=\boldsymbol{\kappa}_{0, G}(x)+\delta \boldsymbol{\kappa}_{G}(x) .
\end{array}
$$


By contrast, the update of the non-linear quantities like boundary rotation vectors is not additive and must consider the multiplicative nature of rotations. The update is as follows: in the first step, the Rodrigues formula (3) is used to determine the changes of boundary rotation matrices, $\delta \mathbf{R}^{0}, \delta \mathbf{R}^{L}$, from $\delta \boldsymbol{\vartheta}^{0}$ and $\delta \boldsymbol{\vartheta}^{L}$. In the next step, improved boundary rotation matrices are obtained by the matrix multiplication:

$$
\mathbf{R}^{0}=\delta \mathbf{R}^{0} \mathbf{R}_{0}^{0}, \quad \mathbf{R}^{L}=\delta \mathbf{R}^{L} \mathbf{R}_{0}^{L}
$$

Finally, the Spurrier algorithm [21] is used to extract new boundary rotational vectors $\boldsymbol{\vartheta}_{g}^{0}$ and $\boldsymbol{\vartheta}_{g}^{L}$ from $\mathbf{R}^{0}$ and $\mathbf{R}^{L}$.

Once the primary unknowns have been determined, the kinematic vectors are obtained by the integration. We first determine $\delta \boldsymbol{\vartheta}_{g}$ using equation (48)

$$
\delta \boldsymbol{\vartheta}_{g}(x)=\delta \boldsymbol{\vartheta}_{g}^{0}+\int_{0}^{x} \mathbf{R}_{0}(\xi) \delta \boldsymbol{\kappa}_{G}(\xi) d \xi
$$

Then we evaluate $\delta \mathbf{R}(x)$ from $\delta \boldsymbol{\vartheta}_{g}(x)$ by the Rodrigues formula, multiply $\delta \mathbf{R}(x)$ and $\mathbf{R}(x)$, and (if needed) extract $\boldsymbol{\vartheta}_{g}(x)$ by the Spurrier algorithm. With the rotation $\mathbf{R}(x)$ being known, $\boldsymbol{r}_{g}(x)$ is obtained from (6) by the integration.

\section{Numerical solution of linearized equations}

Although the first variations of the governing equations (69) are linear functionals, they do not appear in the form of a linear combination of the primary unknowns $\delta \boldsymbol{\gamma}_{G}(x)$ and $\delta \boldsymbol{\kappa}_{G}(x)$. That is why they are usually too demanding to be solved analytically. The discretization of the problem is shown in the sequel.

Functions $\boldsymbol{\gamma}_{G}(x)$ and $\boldsymbol{\kappa}_{G}(x)$ are replaced by the set of their unknown values $\gamma_{G}^{p}$ and $\boldsymbol{\kappa}_{G}^{p}$ at discrete points identified by $x_{p} \in[0, L] ; p=1,2, \ldots, N$, and interpolated by the set of $N$ interpolation functions $I_{p}(x)$

$$
\begin{aligned}
\boldsymbol{\gamma}_{G}(x) & =I_{p}(x) \boldsymbol{\gamma}_{G}^{p} \\
\boldsymbol{\kappa}_{G}(x) & =I_{p}(x) \boldsymbol{\kappa}_{G}^{p}
\end{aligned}
$$

The repeated index $p$ is the summation index. Points $x_{p}$ are called 'interpolation points'.

We are now able to rewrite the integrals containing $\delta \boldsymbol{\kappa}_{G}$ and $\delta \boldsymbol{\gamma}_{G}$ as the linear 
forms in $\delta \boldsymbol{\gamma}_{G}^{p}$ and $\delta \boldsymbol{\kappa}_{G}^{p}(p=1,2, \ldots, N)$ :

$$
\begin{aligned}
\int_{0}^{x} \mathbf{R}(\xi) \delta \boldsymbol{\kappa}_{G}(\xi) d \xi & =\int_{0}^{x} I_{p}(\xi) \mathbf{R}(\xi) d \xi \delta \boldsymbol{\kappa}_{G}^{p}=\mathbf{W}_{p}(x) \delta \boldsymbol{\kappa}_{G}^{p} \\
\int_{0}^{x} \mathbf{R}(\xi) \delta \boldsymbol{\gamma}_{G}(\xi) d \xi & =\int_{0}^{x} I_{p}(\xi) \mathbf{R}(\xi) d \xi \delta \boldsymbol{\gamma}_{G}^{p}=\mathbf{W}_{p}(x) \delta \boldsymbol{\gamma}_{G}^{p}
\end{aligned}
$$

By introducing the interpolation for $\boldsymbol{\gamma}_{G}(x)$ and $\boldsymbol{\kappa}_{G}(x)$, we substitute a continuum problem with a discrete one, in which $2 N$ discrete vectors, $\boldsymbol{\gamma}_{G}^{p}$ and $\boldsymbol{\kappa}_{G}^{p}$, and not functions $\boldsymbol{\gamma}_{G}(x)$ and $\boldsymbol{\kappa}_{G}(x)$, are unknowns of the problem.

Once the unknown functions are discretized, it is also reasonable for the algebraic equations (31)-(32) to be discretized. This is achieved by satisfying equations (31)-(32) at $N$ pre-selected discrete points $x_{q} \in[0, L] ; q=1, \ldots$, $N$, not necessarily coincidental with the interpolation points $x_{p} ; p=1, \ldots$, $N$. After such a collocation-type discretization is made, a set of $2 N$ equations is obtained, which needs to be solved for $2 N$ unknowns $\boldsymbol{\gamma}_{G}^{p}$ and $\boldsymbol{\kappa}_{G}^{p}(p, q=1$, $\ldots, N)$ :

$$
\begin{aligned}
\mathbf{R} \boldsymbol{N}_{G}^{C}\left(x_{q}\right)-\boldsymbol{N}_{g}\left(x_{q}\right) & =\mathbf{0} \\
\mathbf{R} \boldsymbol{M}_{G}^{C}\left(x_{q}\right)-\boldsymbol{M}_{g}\left(x_{q}\right) & =\mathbf{0} .
\end{aligned}
$$

The resulting system of discrete governing equations (74)-(75) and (33)-(38) consists of $2 N+6$ non-linear equations of a beam element, where $N$ denotes the number of the discretization points. The unknowns of the problem are $\boldsymbol{r}_{g}^{0}$, $\boldsymbol{\vartheta}_{g}^{0}, \boldsymbol{N}_{g}^{0}, \boldsymbol{M}_{g}^{0}, \boldsymbol{r}_{g}^{L}, \boldsymbol{\vartheta}_{g}^{L}, \boldsymbol{\gamma}_{G}^{p}$, and $\boldsymbol{\kappa}_{G}^{p}(p=1,2, \ldots, N)$.

After the discretization has been completed and equations (72) and (73) employed, the left-hand sides of equations (69) take the following forms:

$$
\begin{aligned}
\delta \boldsymbol{f}_{1}\left(x_{q}\right)= & -\mathbf{S}\left(\mathbf{R}\left(x_{q}\right) \boldsymbol{N}_{G}^{C}\left(x_{q}\right)\right) \delta \boldsymbol{\vartheta}_{g}^{0}-\mathbf{S}\left(\mathbf{R}\left(x_{q}\right) \boldsymbol{N}_{G}^{C}\left(x_{q}\right)\right) \mathbf{W}_{p}\left(x_{q}\right) \delta \boldsymbol{\kappa}_{G}^{p} \\
& +I_{p}\left(x_{q}\right) \mathbf{R}\left(x_{q}\right) \mathbf{C}_{\gamma \gamma}\left(x_{q}\right) \delta \boldsymbol{\gamma}_{G}^{p}+I_{p}\left(x_{q}\right) \mathbf{R}(x) \mathbf{C}_{\gamma \kappa}\left(x_{q}\right) \delta \boldsymbol{\kappa}_{G}^{p}-\delta \boldsymbol{N}_{g}^{0} \\
\delta \boldsymbol{f}_{2}\left(x_{q}\right)= & -\mathbf{S}\left(\mathbf{R}\left(x_{q}\right) \boldsymbol{M}_{G}^{C}\left(x_{q}\right)\right) \delta \boldsymbol{\vartheta}_{g}^{0}-\mathbf{S}\left(\mathbf{R}\left(x_{q}\right) \boldsymbol{M}_{G}^{C}\left(x_{q}\right)\right) \mathbf{W}_{p}\left(x_{q}\right) \delta \boldsymbol{\kappa}_{G}^{p} \\
& +I_{p}\left(x_{q}\right) \mathbf{R}\left(x_{q}\right) \mathbf{C}_{\kappa \gamma}\left(x_{q}\right) \delta \boldsymbol{\gamma}_{G}^{p}+I_{p}\left(x_{q}\right) \mathbf{R}\left(x_{q}\right) \mathbf{C}_{\kappa \kappa}\left(x_{q}\right) \delta \boldsymbol{\kappa}_{G}^{p}-\delta \boldsymbol{M}_{g}^{0} \\
& +\mathbf{S}\left(\int_{0}^{x_{q}} \mathbf{R}\left(\boldsymbol{\gamma}_{G}-\boldsymbol{c}_{G}\right) d \xi\right) \delta \boldsymbol{N}_{g}^{0}+\int_{0}^{x_{q}} \mathbf{S}\left(\boldsymbol{N}_{g}\right) \mathbf{S}\left(\mathbf{R}\left(\boldsymbol{\gamma}_{G}-\boldsymbol{c}_{G}\right)\right) d \xi \delta \boldsymbol{\vartheta}_{g}^{0} \\
& +\int_{0}^{x_{q}} \mathbf{S}\left(\boldsymbol{N}_{g}\right) \mathbf{S}\left(\mathbf{R}\left(\boldsymbol{\gamma}_{G}-\boldsymbol{c}_{G}\right)\right) \mathbf{W}_{p} d \xi \delta \boldsymbol{\kappa}_{G}^{p}-\int_{0}^{x_{q}} I_{p} \mathbf{S}\left(\boldsymbol{N}_{g}\right) \mathbf{R} d \xi \delta \boldsymbol{\gamma}_{G}^{p} \\
\delta \boldsymbol{f}_{3}= & \delta \boldsymbol{r}_{g}^{L}-\delta \boldsymbol{r}_{g}^{0}+\int_{0}^{L} \mathbf{S}\left(\mathbf{R}\left(\boldsymbol{\gamma}_{G}-\boldsymbol{c}_{G}\right)\right) d x \delta \boldsymbol{\vartheta}_{g}^{0} \\
& +\int_{0}^{L} \mathbf{S}\left(\mathbf{R}\left(\boldsymbol{\gamma}_{G}-\boldsymbol{c}_{G}\right)\right) \mathbf{W}_{p} d x \delta \boldsymbol{\kappa}_{G}^{p}-\mathbf{W}_{p}(L) \delta \boldsymbol{\gamma}_{G}^{p} \\
\delta \boldsymbol{f}_{4}= & \delta \boldsymbol{\vartheta}_{g}^{L}-\delta \boldsymbol{\vartheta}_{g}^{0}-\mathbf{W}_{p}(L) \delta \boldsymbol{\kappa}_{G}^{p} \\
\delta \boldsymbol{f}_{5}= & \delta \boldsymbol{N}_{g}^{0}
\end{aligned}
$$




$$
\begin{aligned}
\delta \boldsymbol{f}_{6}= & \delta \boldsymbol{M}_{g}^{0} \\
\delta \boldsymbol{f}_{7}= & -\delta \boldsymbol{N}_{g}^{0} \\
\delta \boldsymbol{f}_{8}= & \mathbf{S}\left(\int_{0}^{L} \mathbf{R}\left(\boldsymbol{\gamma}_{G}-\boldsymbol{c}_{G}\right) d x\right) \delta \boldsymbol{N}_{g}^{0}+\int_{0}^{L} \mathbf{S}\left(\boldsymbol{N}_{g}\right) \mathbf{S}\left(\mathbf{R}\left(\boldsymbol{\gamma}_{G}-\boldsymbol{c}_{G}\right)\right) d x \delta \boldsymbol{\vartheta}_{g}^{0} \\
& -\delta \boldsymbol{M}_{g}^{0}-\int_{0}^{L} I_{p} \mathbf{S}\left(\boldsymbol{N}_{g}\right) \mathbf{R} d x \delta \boldsymbol{\gamma}_{G}^{p} \\
& +\int_{0}^{L} \mathbf{S}\left(\boldsymbol{N}_{g}\right) \mathbf{S}\left(\mathbf{R}\left(\boldsymbol{\gamma}_{G}-\boldsymbol{c}_{G}\right)\right) \mathbf{W}_{p} d x \delta \boldsymbol{\kappa}_{G}^{p}
\end{aligned}
$$

The matrix coefficients at the variations of the primary unknowns are placed into the tangent stiffness matrix of the element, $\mathbf{K}$. The details of the matrix coefficients are presented in Appendix A.

\section{Algorithm}

We now state the details of the computational algorithm for an initially (naturally) curved and twisted beam in which the cross-sections are orthogonal to the line of centroids in the undeformed configuration. We assume that the interpolation points, $x_{p}$, coincide with the collocation points, $x_{q}$. Then $I_{p}\left(x_{q}\right)=0$ if $p \neq q$ and $I_{p}\left(x_{p}\right)=1$.

- Data:

○ element length $L$;

○ discrete points $x_{p} \in[0, L], p=1, \ldots, N$;

○ interpolation functions $I_{p}(x)$;

○ initial kinematics of the beam $\boldsymbol{r}(x), \boldsymbol{\vartheta}(x)$;

○ boundary loads $\boldsymbol{S}^{0}, \boldsymbol{P}^{0}, \boldsymbol{S}^{L}, \boldsymbol{P}^{L}$;

○ integrals of external distributed force and moment: $\tilde{\boldsymbol{n}}(x)=\int_{0}^{x} \boldsymbol{n} d \xi$, $\tilde{\boldsymbol{m}}(x)=\int_{0}^{x} \boldsymbol{m} d \xi$.

- Evaluate auxiliary functions

$$
\begin{aligned}
\mathbf{R}(x) & =\text { Rodrigues }(\boldsymbol{\vartheta}(x)) \\
\mathbf{R}_{\mathrm{S}}^{(1)}(x) & =\mathbf{S}\left(\mathbf{R}(x)\left[\begin{array}{l}
1 \\
0 \\
0
\end{array}\right]\right) \\
\mathbf{N}(x) & =-\mathbf{S}(\tilde{\boldsymbol{n}}(x))
\end{aligned}
$$

and integrals

$$
\mathbf{W}_{p}(x)=\int_{0}^{x} I_{p} \mathbf{R} d \xi
$$




$$
\begin{aligned}
\mathbf{I}_{I \mathrm{NR}}^{p}(x) & =\int_{0}^{x} I_{p} \mathbf{N R} d \xi \\
\mathbf{I}_{\mathrm{R}}(x) & =\int_{0}^{x} \mathbf{R}_{\mathrm{S}}^{(1)} d \xi \\
\mathbf{I}_{\mathrm{NR}}(x) & =\int_{0}^{x} \mathbf{N} \mathbf{R}_{\mathrm{S}}^{(1)} d \xi \\
\mathbf{I}_{\mathrm{RW}}^{p}(x) & =\int_{0}^{x} \mathbf{R}_{\mathrm{S}}^{(1)} \mathbf{W}_{p} d \xi \\
\mathbf{I}_{\mathrm{NRW}}^{p}(x) & =\int_{0}^{x} \mathbf{N R}_{\mathrm{S}}^{(1)} \mathbf{W}_{p} d \xi \\
\mathbf{I}_{\mathrm{R} n}(x) & =\int_{0}^{x} \mathbf{R}_{\mathrm{S}}^{(1)} \tilde{\boldsymbol{n}} d \xi .
\end{aligned}
$$

- Evaluate the tangent stiffness matrix $\mathbf{K}$ (see Appendix B).

- Evaluate the right hand side (see Appendix C).

- Solve the system of linear equations $\mathrm{K} \Delta \boldsymbol{y}=-\boldsymbol{f}$ for $\Delta \boldsymbol{y}$

$$
\Delta \boldsymbol{y}=\left[{ }_{\Delta} \boldsymbol{r}^{0} \Delta \boldsymbol{\vartheta}^{0}{ }_{\Delta} \boldsymbol{r}^{L} \Delta \boldsymbol{\vartheta}^{L} \Delta \boldsymbol{N}^{0} \Delta \boldsymbol{M}^{0} \Delta \boldsymbol{\kappa}^{1} \ldots \Delta \boldsymbol{\kappa}^{N} \Delta \boldsymbol{\gamma}^{1} \ldots \Delta \boldsymbol{\gamma}^{N}\right]^{T}
$$

add the corrections to the initial values

$$
\begin{aligned}
\boldsymbol{r}(0) & =\boldsymbol{r}(0)+\Delta \boldsymbol{r}^{0} \\
\boldsymbol{r}(L) & =\boldsymbol{r}(L)+\Delta \boldsymbol{r}^{L} \\
\boldsymbol{N}(0) & =\Delta \boldsymbol{N}^{0} \\
\boldsymbol{M}(0) & =\Delta \boldsymbol{M}^{0} \\
\boldsymbol{\kappa}\left(x_{p}\right) & =\Delta \boldsymbol{\kappa}^{p}, \quad p=1, \ldots, N \\
\boldsymbol{\gamma}\left(x_{p}\right) & =\Delta \boldsymbol{\gamma}^{p}, \quad p=1, \ldots, N
\end{aligned}
$$

and extract the boundary rotations:

$$
\begin{aligned}
\boldsymbol{\vartheta}^{0} & =\text { Spurrier }\left(\text { Rodrigues }\left(\Delta \boldsymbol{\vartheta}^{0}\right) \mathbf{R}(0)\right) \\
\boldsymbol{\vartheta}^{L} & =\text { Spurrier }\left(\text { Rodrigues }\left(\Delta \boldsymbol{\vartheta}^{L}\right) \mathbf{R}(L)\right) .
\end{aligned}
$$

- Evaluate the remaining quantities

$$
\begin{aligned}
\Delta \boldsymbol{\vartheta}(x) & =\Delta \boldsymbol{\vartheta}^{0}+\mathbf{W}_{p}(x) \Delta \boldsymbol{\kappa}^{p} \\
\Delta \mathbf{R}(x) & =\operatorname{Rodrigues}(\Delta \boldsymbol{\vartheta}(x)) \\
\mathbf{R}(x) & =\Delta \mathbf{R}(x) \mathbf{R}(x) \\
\boldsymbol{\Gamma}(x) & =\mathbf{R}(x)\left(I_{p}(x) \boldsymbol{\gamma}_{G}^{p}-\left[\begin{array}{lll}
1 & 0 & 0
\end{array}\right]^{T}\right) \\
\boldsymbol{r}(x) & =\boldsymbol{r}(0)+\int_{0}^{x} \boldsymbol{\Gamma}(\xi) d \xi \\
\boldsymbol{N}(x) & =\boldsymbol{N}(0)-\tilde{\boldsymbol{n}}(x) \\
\boldsymbol{M}(x) & =\boldsymbol{M}(0)+\int_{0}^{L} \mathbf{S}(\boldsymbol{\Gamma}) \boldsymbol{N} d x-\tilde{\boldsymbol{m}}(x) .
\end{aligned}
$$




\section{$9 \quad$ Numerical examples}

We present several numerical examples in order to demonstrate the performance and accuracy of the proposed approach. To enable the comparison with theoretical results, only a linear elastic material is employed in all numerical examples. The operators $\mathcal{C}_{N}$ and $\mathcal{C}_{M}$ in (13)-(14) are taken to be diagonal, so that the relationships between stress-resultants and strain measures are given by

$$
\boldsymbol{N}_{G}^{C}=\left[\begin{array}{ccc}
E A_{1} & 0 & 0 \\
0 & G A_{2} & 0 \\
0 & 0 & G A_{3}
\end{array}\right] \boldsymbol{\gamma}_{G}, \quad \boldsymbol{M}_{G}^{C}=\left[\begin{array}{ccc}
G J_{1} & 0 & 0 \\
0 & E J_{2} & 0 \\
0 & 0 & E J_{3}
\end{array}\right] \boldsymbol{\kappa}_{G} .
$$

$E$ and $G$ denote elastic and shear moduli of material; $A_{1}$ is the cross-sectional area; $J_{1}$ is the torsional inertial moment of the cross-section; $A_{2}$ and $A_{3}$ are the effective shear areas in the principal inertial directions $\vec{G}_{2}$ and $\vec{G}_{3}$ of the cross-section; $J_{2}$ and $J_{3}$ are the bending inertial moments of the cross-section about its principal directions $\vec{G}_{2}$ and $\vec{G}_{3}$.

Most of the integrals in the stiffness matrix can be evaluated analytically for sufficiently simple curved beams. We employed Maple's symbolic library [14] for the evaluation of the integrals. When the beam is not curved, the analytical integration turns out to be more efficient than the numerical integration. When the beam is initially curved, the numerical integration is more advantageous. Therefore, for the beams with the curved initial geometry, the Gaussian numerical integration was used with its order equal to the number of the interpolation points. The numerical examples were performed in the Matlab [13] computing environment.

The accuracy of the present numerical model can be enhanced either by (i) the increase of the number of elements $n_{e}$ and/or (ii) the increase of the number of interpolation points in the element, $N$. Several combinations have been tested and excellent results have been obtained even if using only a single beam element with a low-order interpolation. The element, having two interpolation points, was the element of the lowest order used. We wish to stress that the order of the interpolation functions in the computer program is not limited to any particular value, so elements of any order can be chosen. The choice of the position of the interpolation points is free as well. In the present paper the interpolation points are taken to coincide with the Lobatto integration points when analyzing straight elements, and with the Gaussian integration points for the curved elements.

In order to assess the accuracy of the present model we have made comparisons with the analytical solutions whenever possible. The majority of the analytical 
solutions found in literature employ the beam equations which do not fully agree with the present geometrically exact ones. To make the comparisons possible, we have developed our own analytical solutions that are fully in accord with the present theoretical beam model. A short description of the analytical solution of the linearized equations of the three-dimensional beam is presented in Appendix D.

\subsection{Cantilever beam}

We consider a straight in-plane cantilever, subjected to a point moment at its free end (see Figure 2). The analytical solution [16] of the exact non-linear equations of the beam shows that the beam deforms into a part of a circle. The analytical solution of the linearized equations (see Appendix D) for the deformed shape of the cantilever is the parabola

$$
\begin{aligned}
& \vartheta_{Y}(x)=\frac{M_{Y}}{E J_{2}} x \\
& u_{X}(x)=0 \\
& u_{Z}(x)=\frac{M_{Y}}{2 E J_{2}} x^{2} .
\end{aligned}
$$

We compare our numerical results with the above analytical solution and study the convergence of the solution. We also assess the domain of the validity of the linear theory by comparing the non-linear and the linearized solutions.

We took the following geometric and material properties of the cantilever:

$$
\begin{aligned}
E & =2.1 \cdot 10^{4} \quad G=1.05 \cdot 10^{4} \quad L=100 \\
A_{1} & =20 \quad A_{2}=A_{3}=16 \\
J_{1} & =6.4566 \quad J_{2}=1.6667 \quad J_{3}=666.66 .
\end{aligned}
$$

Four different values of the free-end moment were applied (Table 1). A single

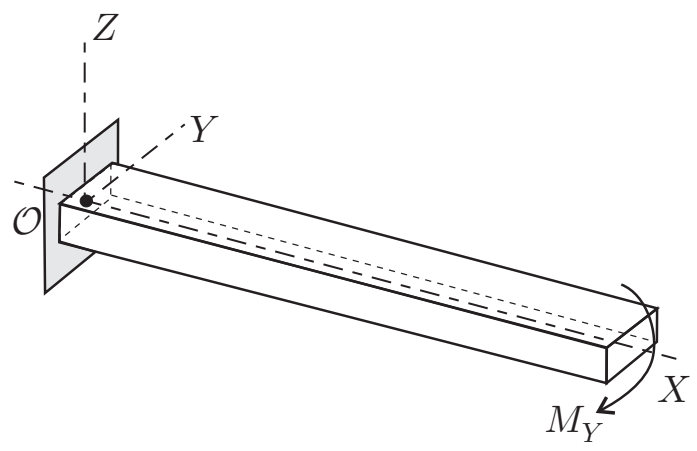

Fig. 2. The cantilever under free-end moment. 
Table 1

Free-end displacements and rotation under an in-plane point moment. Single element of order $N$.

\begin{tabular}{|c|c|c|c|c|}
\hline$M_{Y}$ & method & $u_{X}$ & $u_{Z}$ & $\vartheta_{Y}$ \\
\hline \multirow[t]{4}{*}{$\overline{M_{Y}=1}$} & 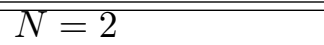 & 0.000000 & 0.142857 & 0.002857 \\
\hline & $N=3$ & 0.000000 & 0.142857 & 0.002857 \\
\hline & exact linear & 0 & 0.142857 & 0.002857 \\
\hline & exact non-lin. [16] & 0.000136 & 0.142857 & 0.002857 \\
\hline \multirow[t]{4}{*}{$M_{Y}=10$} & $\bar{N}=2$ & 0.000000 & 1.428571 & 0.028571 \\
\hline & $N=3$ & 0.000000 & 1.428571 & 0.028571 \\
\hline & exact linear & 0 & 1.428571 & 0.028571 \\
\hline & exact non-lin. [16] & 0.013605 & 1.428474 & 0.028571 \\
\hline \multirow[t]{4}{*}{$M_{Y}=100$} & $N=2$ & 0.000000 & 14.285714 & 0.285714 \\
\hline & $N=3$ & 0.000000 & 14.285714 & 0.285714 \\
\hline & exact linear & 0 & 14.285714 & 0.285714 \\
\hline & exact non-lin. [16] & 1.355002 & 14.188797 & 0.285714 \\
\hline \multirow[t]{4}{*}{$M_{Y}=1000$} & $\bar{N} N=2$ & 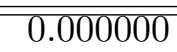 & 142.857143 & 2.857143 \\
\hline & $N=3$ & 0.000000 & 142.857143 & 2.857143 \\
\hline & exact linear & 0 & 142.857143 & 2.857143 \\
\hline & exact non-lin. [16] & 30.706785 & 68.593567 & 2.857143 \\
\hline
\end{tabular}

element and only low-order interpolations along the element were used (linear, $N=2$, and quadratic, $N=3$ ).

In Table 1 the displacements and the rotation of the free end are displayed and compared to the results of the exact values of the non-linear and linearized theories. Note that the analytical solutions of the free-end rotation of linearized and non-linear theories coincide. This is the reason why the present numerical results for the free-end rotation fully agree with the exact ones to all digits for any magnitude of the applied moment.

A complete agreement between the results of exact and numerical linear analyses for the displacements in the $Z$-direction is observed. The displacements in the $Z$-direction are well described by the linearized theory only up to $M_{Y}=100$, when the vertical displacement reaches about $14 \%$ of the beam length.

The displacements in the $X$-direction are predicted to be zero in the linearized theory. The results for the larger moments are thus beyond the scope of the linearized formulation. They clearly show the limitations of linear theories. 


\subsection{Circular arch}

This example shows the ability of the present linear formulation to consider properly the initially curved (non-linear) geometry of the beam. We consider an elastic cantilever beam with the centroidal axis in the form of the circular arc with the central angle $\pi$ and radius $R=5$ (Figure 3 ). The cross-section of the beam is circular with radius $r=0.015$. Material moduli are $E=0.207 \cdot 10^{12}$ and $G=0.95 \cdot 10^{11}$. The cantilever is subjected to the concentrated force of magnitude 100 at its free end. Two load cases are considered: (i) $F_{X}=100$ (here termed the 'in-plane force') and (ii) $F_{Z}=100$ (the 'out-of-plane force').

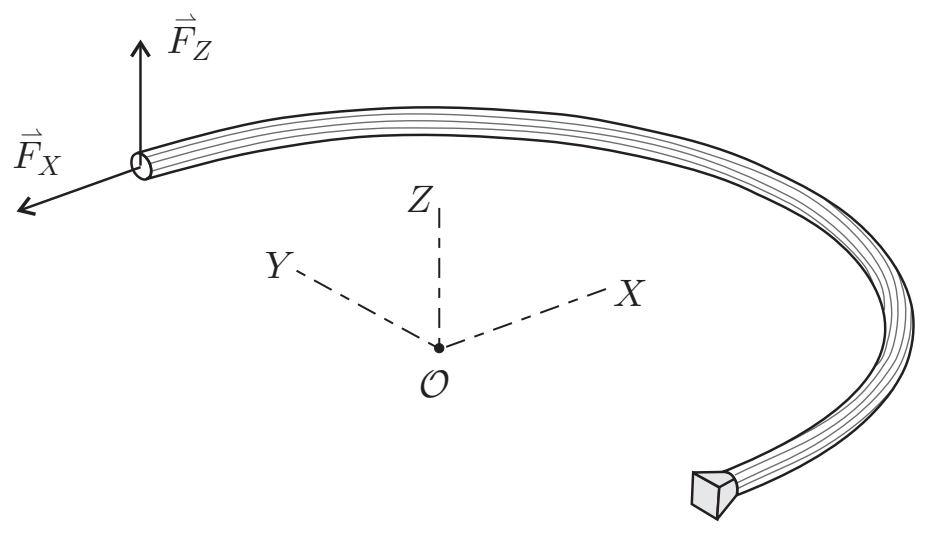

Fig. 3. Circular arch under in-plane and out-of-plane force.

An analytical solution of the problem, in which the effects of shear and axial deflections are neglected, was presented by Tabarrok et al. [22]. Their analytical solution slightly differs from the present analytical solution based on the present theoretical beam model whose equations are presented in Appendix D. Our numerical results for the end displacements and rotations are displayed in Tables 2 and 3 and compared with the analytical values. We used meshes with $n_{e}=1,2,4$ or 8 elements with various degrees of interpolation $(N=2,3, \ldots, 10)$. The tables also display the numerical results of Tabarrok et al. [22] who used their original curved finite elements.

When the in-plane force is applied, the beam remains planar, with the out-ofplane displacements being equal to zero. Analogously, the in-plane displacements are zero in the linearized solution when the out-of-plane force is applied. This is confirmed by the zero numerical values obtained for these displacements. That is why they are not presented in the tables. From Tables 2 and 3 you can observe the excellent agreement between the numerical and the analytical results and the rapid convergence of the results with the increase of the number of elements and/or the number of interpolation points. A single element with cubic interpolation gives the results which are accurate to 4 sig- 
Table 2

In-plane force on circular arch.

\begin{tabular}{|c|c|c|c|c|}
\hline present; $n_{e}$ & $\bar{N}$ & $-u_{X}$ & $-u_{Y}$ & $\Delta \vartheta_{Z}$ \\
\hline \multirow[t]{7}{*}{1} & 2 & 6.4217559 & 3.5101636 & 0.89301087 \\
\hline & 3 & 7.1476279 & 3.0224107 & 0.95558076 \\
\hline & 4 & 7.1560628 & 3.0377089 & 0.95424033 \\
\hline & 5 & 7.1569154 & 3.0374873 & 0.95425549 \\
\hline & 6 & 7.1569334 & 3.0374892 & 0.95425538 \\
\hline & 8 & 7.1569337 & 3.0374892 & 0.95425538 \\
\hline & 10 & 7.1569337 & 3.0374892 & 0.95425538 \\
\hline \multirow[t]{4}{*}{2} & 2 & 7.1168677 & 3.0604562 & 0.95206911 \\
\hline & 3 & 7.1567020 & 3.0373173 & 0.95426701 \\
\hline & 4 & 7.1569301 & 3.0374898 & 0.95425535 \\
\hline & 5 & 7.1569337 & 3.0374892 & 0.95425538 \\
\hline \multirow[t]{3}{*}{4} & 2 & 7.1545258 & 3.0388641 & 0.95414823 \\
\hline & 3 & 7.1569298 & 3.0374867 & 0.95425552 \\
\hline & 4 & 7.1569337 & 3.0374892 & 0.95425538 \\
\hline \multirow[t]{2}{*}{8} & 2 & 7.1567861 & 3.0375758 & 0.95424944 \\
\hline & 3 & 7.1569337 & 3.0374892 & 0.95425538 \\
\hline \multicolumn{2}{|c|}{ Tabarrok et al. $[22]$} & 7.036790 & 3.037488 & 0.95425490 \\
\hline \multicolumn{2}{|c|}{ present analytical } & 7.1568970 & 3.0374892 & 0.95425538 \\
\hline \multicolumn{2}{|c|}{ analytical $[22]$} & $\overline{7.1568990}$ & 3.0374825 & $\overline{0.95425328}$ \\
\hline
\end{tabular}

nificant digits. The comparable accuracy is obtained with 8 linear elements. By increasing the order of the element or the number of the elements at the fixed order of interpolation, the numerical results for $u_{Y}, \Delta \vartheta_{Z}, \Delta \vartheta_{X}$, and $\Delta \vartheta_{Y}$ agree with the analytical ones in all significant digits. The numerical results for $u_{X}$ and $u_{Z}$ are accurate up to 5 and 6 significant digits, respectively. We believe that a slightly lower accuracy of these two results stems from the form of the exact analytical solution. In analytical solutions for $u_{X}$ and $u_{Z}$, the term $x \cos \left(\frac{x}{R}\right)$ occurs. The present numerical method introduces the polynomial interpolation for strains, which is not capable of fully accommodating such a term.

\subsection{Pinched circular ring}

A circular ring with the rectangular cross-section is subjected to two opposite forces. This example has been studied by many authors, see, e.g. [3], [5] and [9]. The ring with the radius of the centroidal axis $R=100$ and the unit width is analyzed here. Two different thicknesses are studied ( $h=1$ and $h=0.1$ ). Elastic modulus is $E=10^{7}$ and Poisson's ratio is $\nu=0.3$. Unit forces are applied as shown in Figure 4. The displacements of the points, marked by $A$ 
and $B$, are evaluated numerically and compared with the analytical solutions.

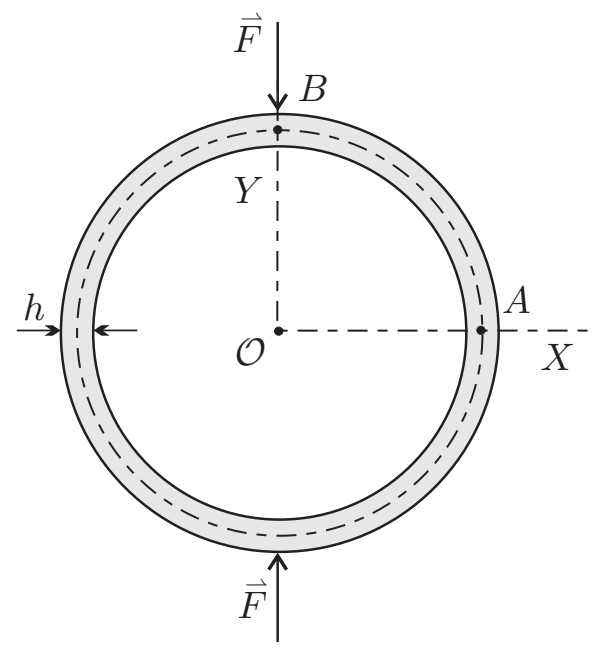

Fig. 4. Circular ring under opposite forces.

It must be stressed that the analytical solution for the deflection of point $B$

Table 3

Out-of-plane force on circular arch: free-end displacements.

\begin{tabular}{|c|c|c|c|c|}
\hline present; $n_{e}$ & $N$ & $u_{Z}$ & $\Delta \vartheta_{X}$ & $\Delta \vartheta_{Y}$ \\
\hline \multirow[t]{7}{*}{1} & 2 & 9.496722 & 0.9425742 & 0.6406139 \\
\hline & 3 & 10.170632 & 0.9973705 & 0.6623125 \\
\hline & 4 & 10.182180 & 0.9969756 & 0.6618477 \\
\hline & 5 & 10.182180 & 0.9969756 & 0.6618477 \\
\hline & 6 & 10.182935 & 0.9969458 & 0.6618529 \\
\hline & 8 & 10.182935 & 0.9969458 & 0.6618529 \\
\hline & 10 & 10.182935 & 0.9969458 & 0.6618529 \\
\hline \multirow[t]{4}{*}{2} & 2 & 10.143260 & 0.9946818 & 0.6600606 \\
\hline & 3 & 10.182929 & 0.9969578 & 0.6618624 \\
\hline & 4 & 10.182933 & 0.9969457 & 0.6618529 \\
\hline & 5 & 10.182935 & 0.9969458 & 0.6618529 \\
\hline \multirow[t]{3}{*}{4} & 2 & 10.180743 & 0.9968347 & 0.6617373 \\
\hline & 3 & 10.182937 & 0.9969459 & 0.6618531 \\
\hline & 4 & 10.182935 & 0.9969458 & 0.6618529 \\
\hline \multirow[t]{2}{*}{8} & 2 & 10.182810 & 0.9969396 & 0.6618456 \\
\hline & 3 & 10.182935 & 0.9969458 & 0.6618529 \\
\hline \multicolumn{2}{|c|}{ Tabarrok et al. $[22]$} & 9.931903 & 0.9931903 & 0.6618526 \\
\hline \multicolumn{2}{|c|}{ present analytical } & 10.182883 & 0.9969458 & 0.6618529 \\
\hline \multicolumn{2}{|c|}{ analytical $[22]$} & 10.182905 & 0.996940 & 0.661850 \\
\hline
\end{tabular}

$n_{e}=$ number of elements, $N=$ order of element. 
in $Y$-direction,

$$
u_{Y}(B)=-0.8927 \frac{F}{E}\left(\frac{R}{h}\right)^{3},
$$

which is usually cited in literature (see, e.g. [3], [9]), does not fully agree with the analytical solution of the present linear formulation which reads

$$
u_{Y}(B)=-0.89267035 \frac{F}{E}\left(\frac{R}{h}\right)^{3}+1.61792022 \frac{F}{E} \frac{R}{h} .
$$

The corresponding analytical solution for the horizontal displacement of point $A$ is

$$
u_{X}(A)=-0.819718634 \frac{F}{E}\left(\frac{R}{h}\right)^{3}+0.53 \frac{F}{E} \frac{R}{h} .
$$

The additional terms are relatively small compared to the first ones. Yet they are important because they indicate the functional dependence of the solution on the thickness of the ring. Table 4 shows the solutions for $h=1$ and $h=0.1$ normalized to $h^{3}$. You can see that the thickness-to-radius ratio of the ring affects the fourth significant digit in the normalized solution.

The present numerical solutions compare well with the analytical ones. The converged solutions are accurate up to 5 significant digits. It has to be noted that the present numerical method gives more accurate results for the thin ring $(h=0.1)$. The values, cited from [3] and [9] were calculated from their solutions normalized with respect to (76).

\begin{tabular}{|c|c|c|c|c|c|c|}
\hline \multirow[b]{2}{*}{ element } & \multirow[b]{2}{*}{$n_{e}$} & \multirow[b]{2}{*}{$N$} & \multicolumn{2}{|c|}{$h=1$} & \multicolumn{2}{|c|}{$h=0.1$} \\
\hline & & & $u_{X}(A)$ & $u_{Y}(B)$ & $u_{X}(A) h^{3}$ & $u_{Y}(B) h^{3}$ \\
\hline \multirow[t]{12}{*}{$\overline{\text { present }}$} & 1 & 2 & 0.0685222 & 0.2369345 & 0.0840586 & 0.0843326 \\
\hline & & 3 & 0.0827712 & 0.0884166 & 0.0819546 & 0.0892119 \\
\hline & & 4 & 0.0819750 & 0.0892848 & 0.0819720 & 0.0892665 \\
\hline & & 6 & 0.0819772 & 0.0892832 & 0.0819719 & 0.0892672 \\
\hline & & 8 & 0.0819772 & 0.0892832 & 0.0819719 & 0.0892672 \\
\hline & 2 & 2 & 0.0731149 & 0.0979452 & 0.0820840 & 0.0889549 \\
\hline & & 3 & 0.0819888 & 0.0892704 & 0.0819717 & 0.0892663 \\
\hline & & 4 & 0.0819772 & 0.0892832 & 0.0819719 & 0.0892672 \\
\hline & & 5 & 0.0819772 & 0.0892832 & 0.0819719 & 0.0892672 \\
\hline & 4 & 2 & 0.0814312 & 0.0898162 & 0.0819786 & 0.0892476 \\
\hline & & 3 & 0.0819773 & 0.0892830 & 0.0819719 & 0.0892672 \\
\hline & & 4 & 0.0819772 & 0.0892832 & 0.0819719 & 0.0892672 \\
\hline \multicolumn{3}{|c|}{ Cho and Lee [3] } & & 0.08925 & & 0.08923 \\
\hline \multicolumn{3}{|c|}{ Kulikov and Plotnikova [9] } & & 0.08852 & & 0.08851 \\
\hline \multicolumn{3}{|c|}{ present analytical } & 0.0819666 & 0.0892509 & 0.0819718 & 0.0892669 \\
\hline
\end{tabular}

Table 4

Displacements of a circular ring. 


\subsection{Twisted cantilever}

An initially twisted beam was presented by MacNeal and Harder [11] as one of standard problems to test the finite-element accuracy. The objective of the test is to prove that the finite element takes the initially non-planar configuration of the beam into account properly. The beam is clamped at one end and subjected to the unit in-plane and the out-of-plane force at the other. The centroidal axis of the beam is straight at the beginning, but the rectangular cross-sections are twisted about the centroidal axis, see Figure 5. The initial twist angle is taken to be a linear function of the arc-length $x$ with its value set to 0 at the clamped end and to $\frac{1}{2} \pi$ at the free end of the beam. The remaining geometric and material characteristics of the beam are:

$$
h=1.1 \quad t=0.32 \quad L=12 \quad E=29 \cdot 10^{6} \quad \nu=0.22 .
$$

The results for the nonzero components of the free-end displacement vector are shown in Table 5 where they are also compared to the analytical results and the theoretical values presented in [11].

The results for the end-point displacements show that the error of the numerical solution is small for any combination of $N$ and $n_{e}$ even with low-order elements. The numerical results for $u_{Z}$ in the first load case and for $u_{Y}$ in the second load case are exact when sufficiently high values for $N$ and/or $n_{e}$ are used. The numerical results for $u_{Y}$ in the first load case and for $u_{Z}$ in the second load case are less accurate due to a different nature of the analytical and the finite-element solution functions (analytical solution is a linear combination of $1, x, x^{2}, x^{3}, \cos \left(\frac{\pi}{L} x\right), \sin \left(\frac{\pi}{L} x\right), x \cos \left(\frac{\pi}{L} x\right)$, and $x \sin \left(\frac{\pi}{L} x\right)$ compared to the polynomial interpolation employed in the numerical solution).

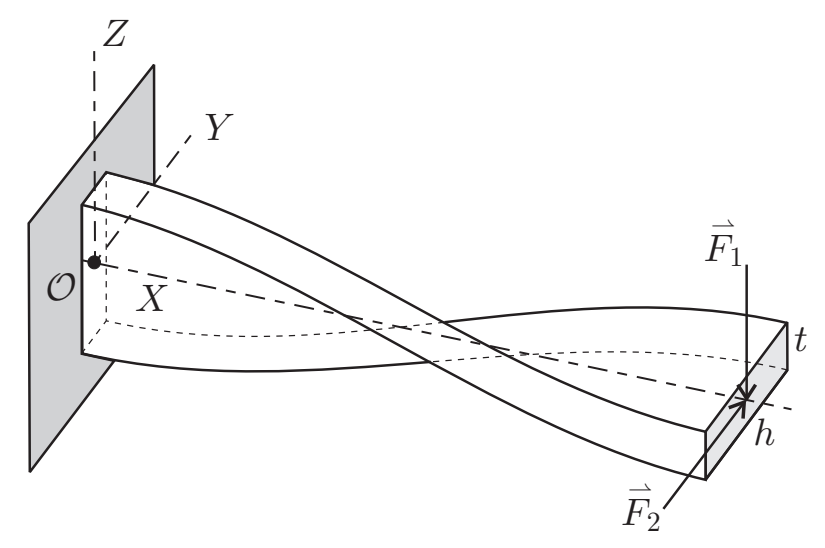

Fig. 5. Twisted beam. Angle of the pretwist is $\pi / 2$. 
Table 5

Free-end displacements of a $\pi / 2$-pretwisted cantilever.

\begin{tabular}{|c|c|c|c|c|c|}
\hline \multirow[b]{2}{*}{$n_{e}$} & \multirow[b]{2}{*}{$N$} & \multicolumn{2}{|c|}{ load case 1} & \multicolumn{2}{|c|}{ load case 2} \\
\hline & & $u_{Y}$ & $u_{Z}$ & $u_{Y}$ & $u_{Z}$ \\
\hline \multirow[t]{5}{*}{1} & 2 & 0.00547212 & 0.00179115 & 0.00184594 & 0.00151703 \\
\hline & 3 & 0.00542141 & 0.00171175 & 0.00171262 & 0.00175084 \\
\hline & 4 & 0.00542933 & 0.00171888 & 0.00171889 & 0.00174950 \\
\hline & 6 & 0.00542932 & 0.00171874 & 0.00171874 & 0.00174962 \\
\hline & 8 & 0.00542932 & 0.00171874 & 0.00171874 & 0.00174962 \\
\hline \multirow[t]{4}{*}{2} & 2 & 0.00542856 & 0.00171726 & 0.00172200 & 0.00173843 \\
\hline & 3 & 0.00542922 & 0.00171869 & 0.00171871 & 0.00174962 \\
\hline & 4 & 0.00542932 & 0.00171874 & 0.00171874 & 0.00174962 \\
\hline & 5 & 0.00542932 & 0.00171874 & 0.00171874 & 0.00174962 \\
\hline \multirow[t]{3}{*}{4} & 2 & 0.00542924 & 0.00171858 & 0.00171892 & 0.00174896 \\
\hline & 3 & 0.00542932 & 0.00171874 & 0.00171874 & 0.00174962 \\
\hline & 4 & 0.00542932 & 0.00171874 & 0.00171874 & 0.00174962 \\
\hline & & 0.005424 & & & 0.001754 \\
\hline & & 0.00542244 & 0.00171874 & 0.00171874 & 0.00174274 \\
\hline
\end{tabular}

\subsection{Twisted circular arch}

In our last example we study the beam, which is both curved and twisted. We assume that the centroidal axis of the beam is a part of the circular arc with the central angle $\pi$ and radius $R=5$ (Figure 6a). The cross-section of the beam is rectangular with width $t=1.1$ and height $h=0.32$. Linear elastic material is employed with material constants $E=29 \cdot 10^{6}$ and $\nu=0.22$. The

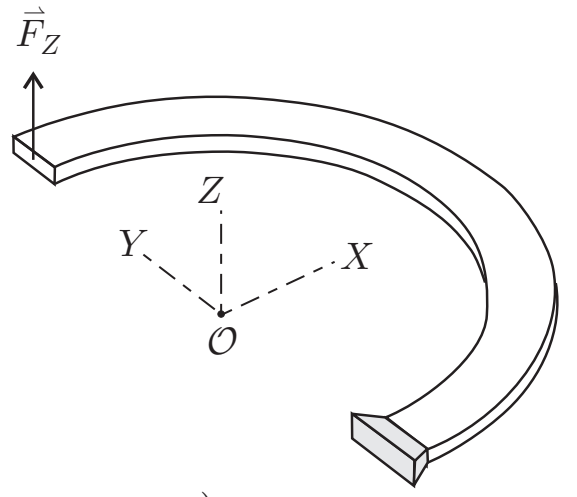

a)

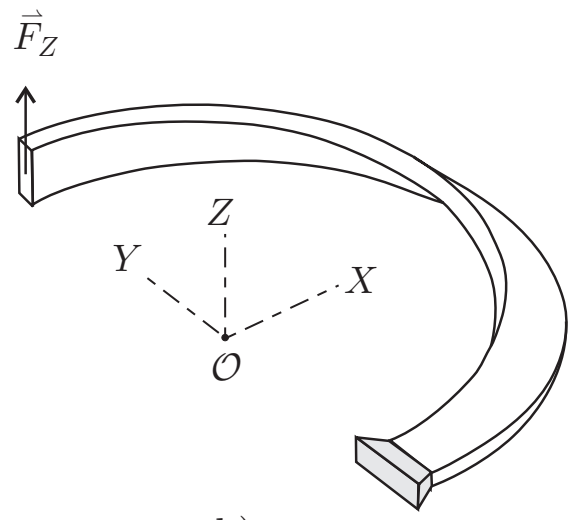

b)

Fig. 6. Circular arch and circular arch with initial $\pi / 2$-pretwist. 
unit concentrated force in the out-of-plane direction is applied at the free end.

The initial twist of the cross-sections is taken to be a linear function of $x$. Six different initial geometries are analyzed, which differ solely in the amplitude of the free-end twist angle, starting from zero in the first case (Figure 6a), to $\frac{\pi}{2}$ in the last, see Figure 6b. Eight quadratic elements are used to model the arch which is sufficient to obtain the results accurate to six significant digits.

Table 6

Free-end displacements of pretwisted circular arches.

\begin{tabular}{crrrr}
\hline \hline free-end twist angle & $u_{X}$ & $u_{Y}$ & $u_{Z}$ & $\|\boldsymbol{u}\|$ \\
\hline 0 & 0.00000000 & 0.00000000 & 0.00730961 & 0.00731 \\
$\pi / 10$ & 0.00030464 & 0.00031675 & 0.00725281 & 0.00727 \\
$2 \pi / 10$ & 0.00058095 & 0.00059091 & 0.00709127 & 0.00714 \\
$3 \pi / 10$ & 0.00080439 & 0.00078730 & 0.00684996 & 0.00694 \\
$4 \pi / 10$ & 0.00095745 & 0.00088402 & 0.00656518 & 0.00669 \\
$\pi / 2$ & 0.00103166 & 0.00087570 & 0.00627795 & 0.00642 \\
\hline \hline
\end{tabular}

Our results of the free-end displacements are shown in Table 6. For the nontwisted beam, the displacement occurs in the $Z$-direction only. The pre-twist of the beam causes the in-plane displacement to emerge also. It increases with the increase of the twist angle and becomes about $16 \%$ of the displacement in the $Z$-direction for the $\frac{\pi}{2}$-twisted beam. At the same time, the amplitude of the out-of-plane displacement decreases; for the $\frac{\pi}{2}$-twisted beam it is reduced by about $14 \%$ with respect to the non-twisted beam. This shows that the beam becomes stiffer with the increase of the pretwist.

Table 7

Analytical results for the free-end displacements of pre-twisted circular arches.

\begin{tabular}{crrrr}
\hline \hline free-end twist angle & $u_{X}$ & $u_{Y}$ & $u_{Z}$ & $\|\boldsymbol{u}\|$ \\
\hline 0 & 0.00000000 & 0.00000000 & 0.00730067 & 0.00730 \\
$\pi / 10$ & 0.00030464 & 0.00031675 & 0.00724387 & 0.00726 \\
$2 \pi / 10$ & 0.00058095 & 0.00059091 & 0.00708233 & 0.00713 \\
$3 \pi / 10$ & 0.00080439 & 0.00078730 & 0.00684102 & 0.00693 \\
$4 \pi / 10$ & 0.00095745 & 0.00088402 & 0.00655625 & 0.00668 \\
$\pi / 2$ & 0.00103166 & 0.00087570 & 0.00626901 & 0.00641 \\
\hline \hline
\end{tabular}

In Table 7 the analytical results are presented. The comparison between Tables 6 and 7 indicates the excellent accuracy of the numerical method. Note 
also slight differences between the numerical and analytical solutions for $u_{Z}$. As in the previous two examples, the differences stem from the form of the exact solution which cannot be described by the low-order polynomial type of discretization with a very high accuracy.

\section{Conclusions}

We presented the governing equations of the consistently linearized geometrically exact spatial beam formulation in which the initial geometric shape of the axis and the twist of the cross-sections are arbitrary. We proposed the finite-element formulation for the solution of the linearized equations. The novelties of the formulation are:

(i) The strain vectors as the only unknown functions are employed, i.e. the formulation is 'strain-based'.

(ii) The linear form of the consistency condition that the equilibrium and the constitutive internal force and moment vectors are equal, is enforced to be satisfied at an arbitrary point of the beam. This conditions are supplemented by the linear form of the boundary and kinematic conditions to yield the complete set of the linearized equations of the spatial beam.

A number of finite elements of different order have been tested by various numerical examples and the results compared to analytical solutions. A rapid convergence and an outstanding accuracy are characteristic for the higherorder elements.

\section{References}

[1] T. M. Atanackovic, V. B. Glavardanov, "Buckling of a twisted and compressed rod", Int. J. Solids Structures 39, 2987-2999, 2002.

[2] J. H. Argyris, "An excursion into large rotations", Comput. Methods Appl. Mech. Eng. 32, 85-155, 1982.

[3] C. Cho, S. W. Lee, "On the assumed strain formulation for geometrically nonlinear analysis", Finite Elements Anal. Design 24, 31-47, 1996.

[4] R. Frisch-Fay, Flexible bars, Butterworths, London, 1962.

[5] M. Ganapathi, B. P. Patel, J. Saravanan, M. Touratier, "Shear flexible curved spline beam element for static analysis", Finite Elements Anal. Design 32, 181202, 1999 . 
[6] A. Ibrahimbegovic, "On the finite element implementation of geometrically nonlinear Reissner's beam theory: 3d curved beam element", Comput. Methods Appl. Mech. Eng. 122, 11-26, 1995.

[7] A. Ibrahimbegovic, "On the choice of finite rotation parameters", Comput. Methods Appl. Mech. Eng. 149, 49-71, 1997.

[8] G. Jelenić, M. Saje, "A kinematically exact space finite strain beam model-finite element formulation by generalized virtual work principle", Comput. Methods Appl. Mech. Eng. 120, 131-161, 1995.

[9] G. M. Kulikov, S. V. Plotnikova, "Non-conventional non-linear two-node hybrid stress-strain curved beam elements", Finite Elements Anal. Design 40, 1333$1359,2004$.

[10] A. Y. T. Leung, "Exact stiffness matrix for twisted helix beam", Finite Elements Anal. Design 9, 23-32, 1991.

[11] R. H. MacNeal, R. L. Harder, "A proposed standard set of problems to test finite element accuracy", Finite Elements Anal. Design 1, 3-20, 1985.

[12] N. Madhusudhana, B. P. Gautham, N. Ganesan, "Buckling behaviour of uniformly and non-uniformly pretwisted beams", Comput. Struct. 54, 551-556, 1995.

[13] The MathWorks, Inc., "MATLAB, Using MATLAB", Natick, http://www. mathworks.com, 1999.

[14] The MathWorks, Inc., "Symbolic Math Toolbox, For Use with MATLAB", Natick, http://www. mathworks.com, 1998.

[15] E. Reissner, "On finite deformation of space-curved beams", J. Appl. Math. Phys. 32, 734-744, 1981.

[16] M. Saje, S. Srpčič, Large deformations of thin curved plane beam of constant initial curvature, Int. J. Mech. Sci. 28, 275-287, 1986.

[17] M. Schulz, F. C. Filippou, "Non-linear spatial Timoshenko beam element with curvature interpolation", Int. J. Numer. Methods Eng. 50, 761-785, 2001.

[18] J. C. Simo, "A finite strain beam formulation. The three-dimensional dynamic problem. Part I", Comput. Methods Appl. Mech. Eng. 49, 55-70, 1985.

[19] J. C. Simo, L. Vu-Quoc, "A three-dimensional finite-strain rod model. Part II: Computational aspects", Comput. Methods Appl. Mech. Eng. 58, 79-116, 1986.

[20] W. M. Smoleński, "Statically and kinematically exact nonlinear theory of rods and its numerical verification", Comput. Methods Appl. Mech. Eng. 178, 89$113,1999$.

[21] R. A. Spurrier, "Comment on Singularity-free extraction of a quaternion from a direction-cosine matrix", J. Spacecraft 15, 255, 1978. 
[22] B. Tabarrok, M. Farshad, H. Yi, "Finite element formulation of spatially curved and twisted rods", Comput. Methods Appl. Mech. Eng. 70, 275-299, 1988.

[23] S. Tang, A. Yu, "Generalized variational principle on nonlinear theory of naturally curved and twisted beams", Appl. Math. Comput. 153, 275-288, 2004.

[24] B. Vratanar, M. Saje, "A consistent equilibrium in a cross-section of an elasticplastic beam", Int. J. Solids Structures 36, 311-337, 1999.

[25] S. P. Timoshenko, J. M. Gere, "Theory of Elastic Stability", McGraw-Hill, New York, 1961.

[26] J. L. Troutman, "Variational Calculus with Elementary Convexity", SpringerVerlag, New York, 1983.

[27] A. Yu, M. Fang, X. Ma, "Theoretical research on naturally curved and twisted beams under complicated loads", Comput. Struct. 80, 2529-2536, 2002.

\section{Appendix A: The tangent stiffness matrix of the element}

The tangent stiffness matrix of the element reads

$$
\mathbf{K}=\left[\begin{array}{cccccccccccc}
\mathbf{0} & \mathbf{F}_{\vartheta}^{1} & \mathbf{0} & \mathbf{0} & -\mathbf{I} & \mathbf{0} & \mathbf{F}_{\kappa, 1}^{1} & \cdots & \mathbf{F}_{\kappa, N}^{1} & \mathbf{F}_{\gamma, 1}^{1} & \cdots & \mathbf{F}_{\gamma, N}^{1} \\
\vdots & \vdots & \vdots & \vdots & \vdots & \vdots & \vdots & \ddots & \vdots & \vdots & \ddots & \vdots \\
\mathbf{0} & \mathbf{F}_{\vartheta}^{N} & \mathbf{0} & \mathbf{0} & -\mathbf{I} & \mathbf{0} & \mathbf{F}_{\kappa, 1}^{N} & \cdots & \mathbf{F}_{\kappa, N}^{N} & \mathbf{F}_{\gamma, 1}^{N} & \cdots & \mathbf{F}_{\gamma, N}^{N} \\
\mathbf{0} & \mathbf{G}_{\vartheta}^{1} & \mathbf{0} & \mathbf{0} & \mathbf{G}_{a}^{1} & -\mathbf{I} & \mathbf{G}_{\kappa, 1}^{1} & \cdots & \mathbf{G}_{\kappa, N}^{1} & \mathbf{G}_{\gamma, 1}^{1} & \cdots & \mathbf{G}_{\gamma, N}^{1} \\
\vdots & \vdots & \vdots & \vdots & \vdots & \vdots & \vdots & \ddots & \vdots & \vdots & \ddots & \vdots \\
\mathbf{0} & \mathbf{G}_{\vartheta}^{N} & \mathbf{0} & \mathbf{0} & \mathbf{G}_{a}^{N} & -\mathbf{l} & \mathbf{G}_{\kappa, 1}^{N} & \cdots & \mathbf{G}_{\kappa, N}^{N} & \mathbf{G}_{\gamma, 1}^{N} & \cdots & \mathbf{G}_{\gamma, N}^{N} \\
-\mathbf{I} & \mathbf{H}_{\vartheta} & \mathbf{I} & \mathbf{0} & \mathbf{0} & \mathbf{0} & \mathbf{H}_{\kappa, 1} & \cdots & \mathbf{H}_{\kappa, N} & \mathbf{H}_{\gamma, 1} & \cdots & \mathbf{H}_{\gamma, N} \\
\mathbf{0} & -\mathbf{l} & \mathbf{0} & \mathbf{I} & \mathbf{0} & \mathbf{0} & \mathbf{H}_{\gamma, 1} & \cdots & \mathbf{H}_{\gamma, N} & \mathbf{0} & \cdots & \mathbf{0} \\
\mathbf{0} & \mathbf{0} & \mathbf{0} & \mathbf{0} & \mathbf{I} & \mathbf{0} & \mathbf{0} & \cdots & \mathbf{0} & \mathbf{0} & \cdots & \mathbf{0} \\
\mathbf{0} & \mathbf{0} & \mathbf{0} & \mathbf{0} & \mathbf{0} & \mathbf{I} & \mathbf{0} & \cdots & \mathbf{0} & \mathbf{0} & \cdots & \mathbf{0} \\
\mathbf{0} & \mathbf{0} & \mathbf{0} & \mathbf{0} & -\mathbf{l} & \mathbf{0} & \mathbf{0} & \cdots & \mathbf{0} & \mathbf{0} & \cdots & \mathbf{0} \\
\mathbf{0} & \mathbf{L}_{\vartheta} & \mathbf{0} & \mathbf{0} & \mathbf{H}_{\vartheta} & -\mathbf{l} & \mathbf{L}_{\kappa, 1} & \cdots & \mathbf{L}_{\kappa, N} & \mathbf{L}_{\gamma, 1} & \cdots & \mathbf{L}_{\gamma, N}
\end{array}\right]
$$


where the $3 \times 3$ block matrices $\mathbf{F}, \mathbf{G}$ and $\mathbf{H}$ are defined by

$$
\begin{aligned}
\mathbf{F}_{\vartheta}^{q}= & -\mathbf{S}\left(\mathbf{R} \mathbf{N}_{G}^{C}\right)_{q} \\
\mathbf{F}_{\kappa, p}^{q}= & \left(I_{p} \mathbf{R} \mathbf{C}_{\gamma \kappa}\right)_{q}-\mathbf{S}\left(\mathbf{R} \mathbf{N}_{G}^{C} \mathbf{W}_{p}\right)_{q} \\
\mathbf{F}_{\gamma, p}^{q}= & \left(I_{p} \mathbf{R} \mathbf{C}_{\gamma \gamma}\right)_{q} \\
\mathbf{G}_{\vartheta}^{q}= & -\mathbf{S}\left(\mathbf{R} \boldsymbol{M}_{G}^{C}\right)_{q}+\int_{0}^{x_{q}} \mathbf{S}\left(\boldsymbol{N}_{g}\right) \mathbf{S}\left(\mathbf{R}\left(\boldsymbol{\gamma}_{G}-\boldsymbol{c}_{G}\right)\right) d \xi \\
\mathbf{G}_{a}^{q}= & \int_{0}^{x_{q}} \mathbf{S}\left(\mathbf{R}\left(\boldsymbol{\gamma}_{G}-\boldsymbol{c}_{G}\right)\right) d \xi \\
\mathbf{G}_{\kappa, p}^{q}= & \left(I_{p} \mathbf{R} \mathbf{C}_{\kappa \kappa}\right)_{q}-\mathbf{S}\left(\mathbf{R} \boldsymbol{M}_{G}^{C} \mathbf{W}_{p}\right)_{q} \\
& +\int_{0}^{x_{q}} \mathbf{S}\left(\boldsymbol{N}_{g}\right) \mathbf{S}\left(\mathbf{R}\left(\boldsymbol{\gamma}_{G}-\boldsymbol{c}_{G}\right)\right) \mathbf{W}_{p} d \xi \\
\mathbf{G}_{\gamma, p}^{q}= & \left(I_{p} \mathbf{R} \mathbf{C}_{\kappa \gamma}\right)_{q}-\int_{0}^{x_{q}} I_{p} \mathbf{S}\left(\mathbf{N}_{g}\right) \mathbf{R} d \xi \\
\mathbf{H}_{\vartheta}= & \int_{0}^{L} \mathbf{S}\left(\mathbf{R}\left(\boldsymbol{\gamma}_{G}-\boldsymbol{c}_{G}\right)\right) d x \\
\mathbf{H}_{\kappa, p}= & \int_{0}^{L} \mathbf{S}\left(\mathbf{R}\left(\boldsymbol{\gamma}_{G}-\boldsymbol{c}_{G}\right)\right) \mathbf{W}_{p} d x \\
\mathbf{H}_{\gamma, p}= & -\mathbf{W}_{p}(L) \\
\mathbf{L}_{\vartheta}= & \int_{0}^{L} \mathbf{S}\left(\boldsymbol{N}_{g}\right) \mathbf{S}\left(\mathbf{R}\left(\gamma_{G}-\boldsymbol{c}_{G}\right)\right) d x \\
\mathbf{L}_{\kappa, p}= & \int_{0}^{L} \mathbf{S}\left(\boldsymbol{N}_{g}\right) \mathbf{S}\left(\mathbf{R}\left(\gamma_{G}-\boldsymbol{c}_{G}\right)\right) \mathbf{W}_{p} d x \\
\mathbf{L}_{\gamma, p}= & -\int_{0}^{L} I_{p} \mathbf{S}\left(\boldsymbol{N}_{g}\right) \mathbf{R} d x .
\end{aligned}
$$

The subscript ' $q$ ' marks that the term is evaluated at $x_{q}$.

When the cross-sections are orthogonal to the line of centroids at the undeformed configuration, the block matrices $\mathbf{F}, \mathbf{G}, \mathbf{H}$, and $\mathbf{L}$ assume considerably simpler forms

$$
\begin{aligned}
\mathbf{F}_{\vartheta}^{q} & =\mathbf{0} \\
\mathbf{F}_{\kappa, p}^{q} & =\left(I_{p} \mathbf{R C}_{\gamma \kappa}\right)_{q} \\
\mathbf{F}_{\gamma, p}^{q} & =\left(I_{p} \mathbf{R C}_{\gamma \gamma}\right)_{q} \\
\mathbf{G}_{\vartheta}^{q} & =\int_{0}^{x_{q}} \mathbf{S}\left(\mathbf{N}_{g}\right) \mathbf{R}_{\mathrm{S}}^{(1)} d \xi \\
\mathbf{G}_{a}^{q} & =\int_{0}^{x_{q}} \mathbf{R}_{\mathrm{S}}^{(1)} d \xi \\
\mathbf{G}_{\kappa, p}^{q} & =\left(I_{p} \mathbf{R} \mathbf{C}_{\kappa \kappa}\right)_{q}+\int_{0}^{x_{q}} \mathbf{S}\left(\mathbf{N}_{g}\right) \mathbf{R}_{\mathrm{S}}^{(1)} \mathbf{W}_{p} d \xi \\
\mathbf{G}_{\gamma, p}^{q} & =\left(I_{p} \mathbf{R C}_{\kappa \gamma}\right)_{q}-\int_{0}^{x_{q}} I_{p} \mathbf{S}\left(\mathbf{N}_{g}\right) \mathbf{R} d \xi \\
\mathbf{H}_{\vartheta} & =\int_{0}^{L} \mathbf{R}_{\mathrm{S}}^{(1)} d x
\end{aligned}
$$




$$
\begin{aligned}
\mathbf{H}_{\kappa, p} & =\int_{0}^{L} \mathbf{R}_{\mathrm{S}}^{(1)} \mathbf{W}_{p} d x \\
\mathbf{H}_{\gamma, p} & =-\mathbf{W}_{p}(L) \\
\mathbf{L}_{\vartheta} & =\int_{0}^{L} \mathbf{S}\left(\boldsymbol{N}_{g}\right) \mathbf{R}_{\mathrm{S}}^{(1)} d x \\
\mathbf{L}_{\kappa, p} & =\int_{0}^{L} \mathbf{S}\left(\boldsymbol{N}_{g}\right) \mathbf{R}_{\mathrm{S}}^{(1)} \mathbf{W}_{p} d x \\
\mathbf{L}_{\gamma, p} & =-\int_{0}^{L} I_{p} \mathbf{S}\left(\boldsymbol{N}_{g}\right) \mathbf{R} d x .
\end{aligned}
$$

$\mathbf{R}_{\mathrm{S}}^{(1)}$ is the skew-symmetric matrix $\mathbf{S}\left(\mathbf{R}\left[\begin{array}{l}1 \\ 0 \\ 0\end{array}\right]\right)$.

The column of varied unknowns associated with matrix $\mathbf{K}$ reads

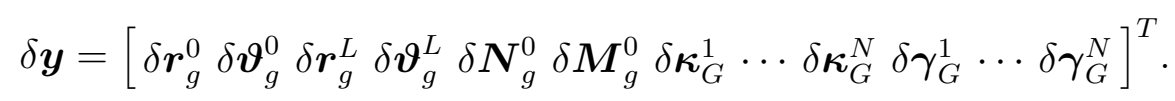

It is obvious from the above expressions and also from Appendix B that the tangent stiffness matrix is non-symmetric. The non-symmetry stems from taking the consistency conditions (15)-(16) as a part of the governing equations. Despite the loss of symmetry and some increase in computational demands an overall benefit is found in materially non-linear problems. Another issue, which is also evident from the above expressions, is the independence of the tangent stiffness matrix on the loading of the beam. That is why the linear theory is not capable of detecting possible critical (bifurcation and limit) points. 
Appendix B: The tangent stiffness matrix of the element for the beam whose cross-sections are orthogonal to the line of centroids at the undeformed configuration

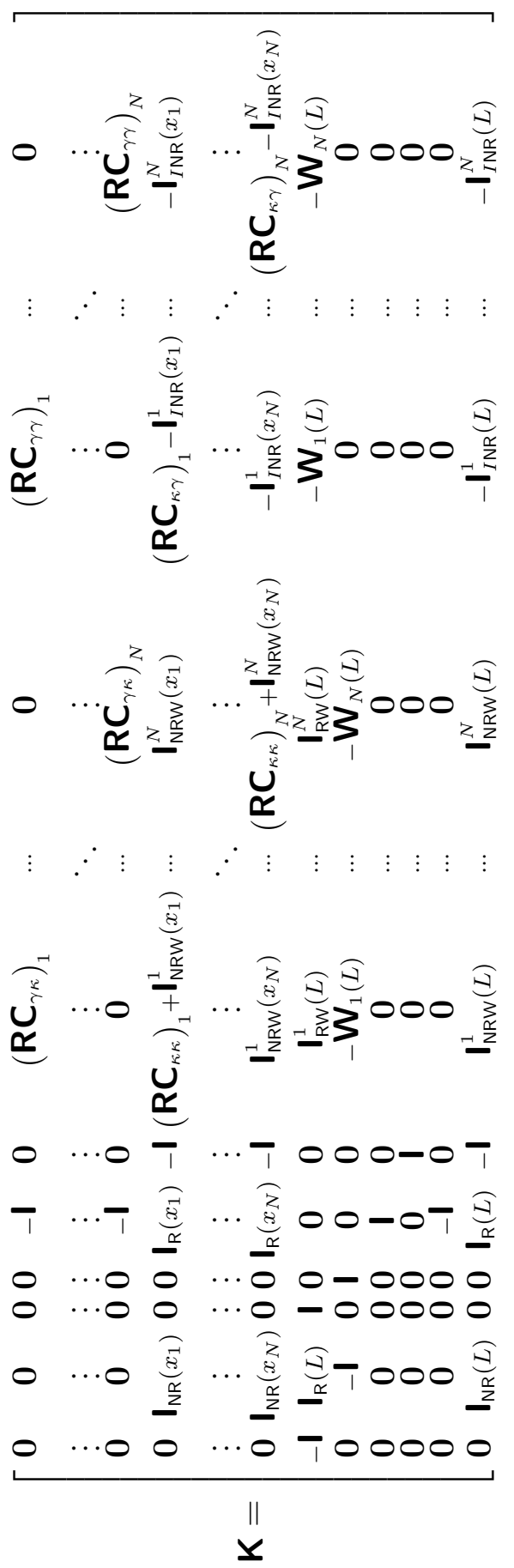


Appendix C: The residual vector of the element for the beam whose cross-sections are orthogonal to the line of centroids at the undeformed configuration

$$
-\boldsymbol{f}=\left[\begin{array}{l}
-\tilde{\boldsymbol{n}}\left(x_{1}\right) \\
\vdots \\
-\tilde{\boldsymbol{n}}\left(x_{N}\right) \\
\mathbf{I}_{\mathrm{R} n}\left(x_{1}\right)-\tilde{\boldsymbol{m}}\left(x_{1}\right) \\
\vdots \\
\mathbf{I}_{\mathrm{R} n}\left(x_{N}\right)-\tilde{\boldsymbol{m}}\left(x_{N}\right) \\
\mathbf{0} \\
\mathbf{0} \\
-\boldsymbol{S}^{0} \\
-\boldsymbol{P}^{0} \\
-\boldsymbol{S}^{L}-\tilde{\boldsymbol{n}}(L) \\
\boldsymbol{P}^{L}+\mathbf{I}_{\mathrm{R} n}(L)-\tilde{\boldsymbol{m}}(L)
\end{array}\right]
$$

\section{Appendix D: Analytical solution}

The present analytical solution assumes the linear material of the beam, initial stress-resultant vectors equal to zero, $\boldsymbol{N}_{g}(x)=\boldsymbol{M}_{g}(x)=\mathbf{0}$, and arbitrary kinematic vectors $\boldsymbol{r}_{g}(x)$ and $\boldsymbol{\vartheta}_{g}(x)$ at the undeformed configuration. The initial strains should satisfy the kinematic equations (6)-(7). We also assume that the beam is subjected only to boundary point loads and moments; i.e., the external distributed loading is disregarded.

We seek the analytical solution of equations (17)-(22). Firstly, the algebraic equations (17)-(18) are eliminated from (17)-(22). The remaining equations (19)-(22) constitute the system of the first order differential equations, which are then linearized as described in Section 6. Because equations (17)-(18) have been eliminated, some of the results in Section 6 cannot be directly applied and the deductions have to be redone.

The linear constitutive law combined with the consistency conditions (17)- 
(18) yields the relationship between the strains and the stress-resultants

$$
\begin{aligned}
\gamma_{G} & =\mathbf{C}_{N}^{-1} \boldsymbol{N}_{G}^{C}=\mathbf{C}_{N}^{-1} \mathbf{R}^{T} \boldsymbol{N}_{g} \\
\boldsymbol{\kappa}_{G} & =\mathbf{C}_{M}^{-1} \boldsymbol{M}_{G}^{C}=\mathbf{C}_{M}^{-1} \mathbf{R}^{T} \boldsymbol{M}_{g} .
\end{aligned}
$$

The variations of $\boldsymbol{\gamma}_{G}$ and $\boldsymbol{\kappa}_{G}$ yield

$$
\begin{aligned}
& \delta \boldsymbol{\gamma}_{G}=\mathbf{C}_{N}^{-1}\left(-\mathbf{R}^{T} \delta \boldsymbol{\Theta} \boldsymbol{N}_{g}+\mathbf{R}^{T} \delta \boldsymbol{N}_{g}\right)=\mathbf{C}_{N}^{-1} \mathbf{R}^{T}\left(\delta \boldsymbol{N}_{g}-\delta \boldsymbol{\vartheta}_{g} \times \boldsymbol{N}_{g}\right) \\
& \delta \boldsymbol{\kappa}_{G}=\mathbf{C}_{M}^{-1}\left(-\mathbf{R}^{T} \delta \boldsymbol{\Theta} \boldsymbol{M}_{g}+\mathbf{R}^{T} \delta \boldsymbol{M}_{g}\right)=\mathbf{C}_{M}^{-1} \mathbf{R}^{T}\left(\delta \boldsymbol{M}_{g}-\delta \boldsymbol{\vartheta}_{g} \times \boldsymbol{M}_{g}\right)
\end{aligned}
$$

The linearization of equations (19)-(20) is straightforward. Considering that $\boldsymbol{n}_{g}=0$ and $\boldsymbol{m}_{g}=0$ and that equation (20) can be replaced by (12), we get

$$
\begin{array}{r}
\delta \boldsymbol{N}_{g}^{\prime}=\mathbf{0} \\
\delta \boldsymbol{M}_{g}^{\prime}+\delta \boldsymbol{r}_{g}^{\prime} \times \boldsymbol{N}_{g}+\boldsymbol{r}_{g}^{\prime} \times \delta \boldsymbol{N}_{g}=\mathbf{0} .
\end{array}
$$

The linearization of equations (21)-(22) follows from (4)-(5). After inserting (79)-(80) into (4)-(5) and rearranging the terms we get

$$
\begin{aligned}
\delta \boldsymbol{r}_{g}^{\prime}-\delta \boldsymbol{\vartheta}_{g} \times \boldsymbol{r}_{g}^{\prime} & =\mathbf{R C}_{N}^{-1} \mathbf{R}^{T}\left(\delta \boldsymbol{N}_{g}-\delta \boldsymbol{\vartheta}_{g} \times \boldsymbol{N}_{g}\right) \\
\delta \boldsymbol{\vartheta}_{g}^{\prime} & =\mathbf{R C}_{M}^{-1} \mathbf{R}^{T}\left(\delta \boldsymbol{M}_{g}-\delta \boldsymbol{\vartheta}_{g} \times \boldsymbol{M}_{g}\right)
\end{aligned}
$$

The solution of linearized equations (81)-(84) is sought at the initially unloaded and undeformed configuration of the beam which gives the final set of the linearized equations for the unknowns $\delta \boldsymbol{N}_{g}, \delta \boldsymbol{M}_{g}, \delta \boldsymbol{r}_{g}$ and $\delta \boldsymbol{\vartheta}_{g}$ :

$$
\begin{aligned}
\delta \boldsymbol{N}_{g}^{\prime} & =\mathbf{0} \\
\delta \boldsymbol{M}_{g}^{\prime}+\boldsymbol{r}_{g}^{\prime} \times \delta \boldsymbol{N}_{g} & =\mathbf{0} \\
\delta \boldsymbol{r}_{g}^{\prime}-\delta \boldsymbol{\vartheta}_{g} \times \boldsymbol{r}_{g}^{\prime}-\mathbf{R C}_{N}^{-1} \mathbf{R}^{T} \delta \boldsymbol{N}_{g} & =\mathbf{0} \\
\delta \boldsymbol{\vartheta}_{g}^{\prime}-\mathbf{R C}_{M}^{-1} \mathbf{R}^{T} \delta \boldsymbol{M}_{g} & =\mathbf{0} .
\end{aligned}
$$

Vectors $\boldsymbol{r}_{g}^{\prime}$ and $\boldsymbol{\vartheta}_{g}$ and the related rotation matrix $\mathbf{R}$ in (85)-(88) represent the given data describing the undeformed configuration. The solution of (85)-(88) must satisfy the kinematic and force boundary conditions.

Note that the actual solution depends on the initial geometry and the boundary conditions. It is useful to observe that the system of differential equations (85)-(88) can be separated into two sets. Equations (85)-(86) are solved first for $\delta \boldsymbol{N}_{g}$ and $\delta \boldsymbol{M}_{g}$. After $\delta \boldsymbol{N}_{g}$ and $\delta \boldsymbol{M}_{g}$ have been obtained, they are inserted into equations (87)-(88), which are then conveniently solved for $\delta \boldsymbol{r}_{g}$ and $\delta \boldsymbol{\vartheta}_{g}$ by Mathematica. 